MATHEMATICS OF COMPUTATION

Volume 71, Number 239, Pages 1043-1074

S 0025-5718(02)01446-1

Article electronically published on January 17, 2002

\title{
HERMITE INTERPOLATION OF NONSMOOTH FUNCTIONS PRESERVING BOUNDARY CONDITIONS
}

\author{
V. GIRAULT AND L. R. SCOTT
}

\begin{abstract}
This article is devoted to the construction of a Hermite-type regularization operator transforming functions that are not necessarily $\mathcal{C}^{1}$ into globally $\mathcal{C}^{1}$ finite-element functions that are piecewise polynomials. This regularization operator is a projection, it preserves appropriate first and second order polynomial traces, and it has approximation properties of optimal order. As an illustration, it is used to discretize a nonhomogeneous Navier-Stokes problem, with tangential boundary condition.
\end{abstract}

\section{INTRODUCTION}

The approximation and regularization of functions in low-order Sobolev spaces by Lagrange finite-element spaces has been well studied (cf. [7, 18], [4] and the references therein). The main idea is to define an interpolation operator using local averaging (regularizing) to define nodal values for functions even in $L^{1}$. However, to our knowledge, such constructions have not been carried out for more general interpolants, such as Hermite-type interpolants which involve derivative data. Following [18, 15] and [17] closely, we construct such an interpolant which preserves polynomial nonhomogeneous boundary conditions naturally. We apply this to approximate divergence-free vector fields by divergence-free piecewise polynomials.

Due to our interest in divergence-free, piecewise polynomial vector fields, which can be written (in two dimensions) as the curl of stream functions that are $\mathcal{C}^{1}$ piecewise polynomials on the same mesh [17], we will limit our attention to interpolation by $\mathcal{C}^{1}$ piecewise polynomials as opposed to more general Hermite interpolants. Similarly, we restrict our attention to triangular finite elements in two dimensions, $d=2$. But we assume only that the meshes are nondegenerate, i.e., we do not assume that they are quasi-uniform. Although there are simpler $\mathcal{C}^{1}$ interpolants (such as the Argyris element [6]), in order to apply it to rough functions, or to impose appropriate boundary conditions, it appears that we need to use an interpolant based on the full space of $\mathcal{C}^{1}$ piecewise polynomials, similar to the one introduced in [15]. Averaging is done either on a triangle or on an edge of some triangle, and the key point is that this operator averages the interpolated functions on a segment of the boundary of the domain for each boundary nodal variable. As a consequence, this interpolation operator preserves homogeneous (and suitable polynomial, nonhomogeneous) boundary conditions naturally and is of optimal order in approximation.

Received by the editor October 15, 1999.

2000 Mathematics Subject Classification. Primary 65D05; Secondary 65N15, 65N30.

Key words and phrases. Hermite interpolation, regularization, divergence-zero finite elements, Leray-Hopf lifting. 
Of course, the interpolator can be defined for as broad a class of functions as that of [7] and [4], but in order to preserve homogeneous boundary conditions, it must be specialized to functions smooth enough to have well-defined boundary values.

As an illustration, these finite-element spaces are applied to the discretization of a nonhomogeneous Navier-Stokes equation with a tangential boundary condition. First, we construct a divergence-zero Leray-Hopf lifting (cf. [11, [12, [13]) of the nonhomogeneous boundary data, with support concentrated near the boundary. This generalizes to a Lipschitz-continuous boundary, a result of [20]. Then we interpolate the lifting, discretize the Navier-Stokes problem and analyze its solutions. The lifting we construct and its interpolant are very important. Indeed, in contrast to the original Leray-Hopf lifting, whose gradient grows exponentially with the Reynolds number, our lifting's gradient and the gradient of its interpolant only increase as the square root of the Reynolds number.

This article is organized as follows. In Section 2, we recall some notation and the notion of singular vertices, which plays an important part in the sequel. The nodal degrees of freedom of $\mathcal{C}^{1}$ finite elements are described in Section 3. In Section 4 , these degrees of freedom are transformed by appropriate integrations in order to preserve traces and to be defined for functions that do not belong to $\mathcal{C}^{1}$. The traces of the resulting finite-element spaces are described in Section 5, and the projection and trace-preserving properties of the interpolation operator are established. Section 6 is devoted to a corresponding boundary interpolation operator. Section 7 addresses the interpolation error. Finite-element spaces of $H^{1}$ functions with zero divergence are constructed in Section 8. In Section 9, we construct a divergencefree lifting of a tangential boundary vector with support concentrated near the boundary. This lifting is used in Section 10 for discretizing a nonhomogeneous Navier-Stokes problem.

\section{NotATION}

Let $\Omega \subset \mathbb{R}^{2}$ be a connected, open, bounded domain with a polygonal boundary $\partial \Omega$. We assume that $\partial \Omega$ is Lipschitz-continuous for simplicity, although many of the results presented here can be extended easily to domains with simple slits. Also, we do not assume that $\Omega$ is simply-connected, and we denote by $\Gamma_{i}, 0 \leq i \leq J$, the connected components of $\partial \Omega$. The following definitions of spaces are valid in any dimension $d$. Let $\mathcal{C}^{\infty}(\Omega)$ be the set of functions defined on $\Omega$ and having continuous derivatives of any order, and let $\mathcal{D}(\Omega)$ be functions in $\mathcal{C}^{\infty}(\Omega)$ with compact support in $\Omega$. For any number $p$ with $1 \leq p \leq \infty$ and any integer $l \geq 1$, we denote by $L^{p}(\Omega)$ the completion of the functions in $\mathcal{C}^{\infty}(\Omega)$ such that $\|f\|_{L^{p}(\Omega)}:=\left(\int_{\Omega}|f(x)|^{p} d x\right)^{1 / p}$ is finite, by $W^{l, p}(\Omega)$ the completion of the functions in $\mathcal{C}^{\infty}(\Omega)$ such that $\|f\|_{W^{l, p}(\Omega)}:=\left(\sum_{|\alpha| \leq l}\left\|D^{\alpha} f\right\|_{L^{p}(\Omega)}^{p}\right)^{1 / p}$ is finite, and by $W_{0}^{l, p}(\Omega)$ the completion of the functions in $\mathcal{D}(\Omega)$ such that $\|f\|_{W^{l, p}(\Omega)}$ is finite. Similarly, we define seminorms,

$$
|f|_{W^{l, p}(\Omega)}:=\left(\sum_{|\alpha|=l}\left\|D^{\alpha} f\right\|_{L^{p}(\Omega)}^{p}\right)^{1 / p} .
$$

Here $\alpha=\left(\alpha_{1}, \ldots, \alpha_{d}\right)$ is a multi-index (each $\alpha_{i}$ is a nonnegative integer) with $|\alpha|:=\sum_{i=1}^{d} \alpha_{i}$ and $D^{\alpha}:=\left(\partial / \partial x_{1}\right)^{\alpha_{1}} \ldots\left(\partial / \partial x_{d}\right)^{\alpha_{d}}$. As usual, we denote $W^{l, 2}(\Omega)$ 


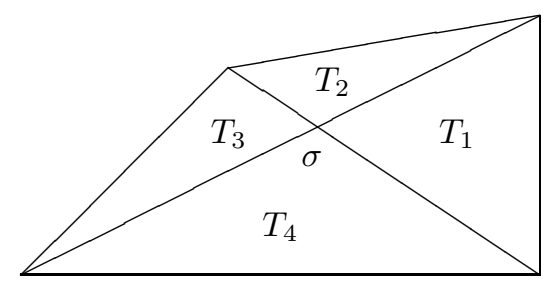

FIgURE 1. Interior singular vertex.

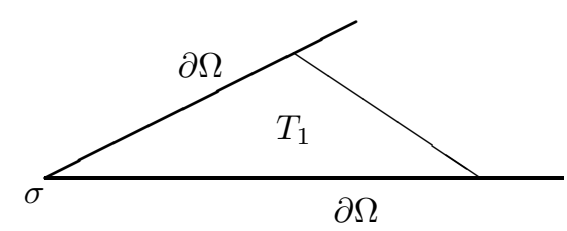

FiguRE 2. Boundary singular vertex: a triangle with two boundary edges.

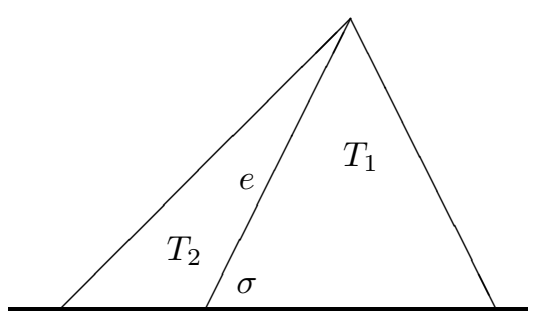

$\partial \Omega$

FigURE 3. Boundary singular vertex where two triangles meet.

and $W_{0}^{l, 2}(\Omega)$ by $H^{l}(\Omega)$ and $H_{0}^{l}(\Omega)$ respectively. For the definition of $W^{l, p}(\Omega)$ for fractional-order $l$, see [1], 10, [14, or [16].

Let $\mathcal{T}_{h}$ be a simplicial subdivision of $\bar{\Omega}$ consisting of triangles with maximum mesh size

$$
h:=\max _{T \in \mathcal{T}_{h}} h_{T}
$$

that is nondegenerate:

$$
\max _{T \in \mathcal{T}_{h}} \frac{h_{T}}{\rho_{T}} \leq \gamma_{0}
$$

with the constant $\gamma_{0}$ independent of $h$. Here $T$ denotes a triangle, $h_{T}$ denotes the diameter of $T$ and $\rho_{T}$ denotes the radius of the ball inscribed in $T$. Note that we do not assume that all triangles $T$ are of comparable size (that is, the mesh need not be quasi-uniform).

In the sequel, we shall need to distinguish between nonsingular and singular vertices of $\mathcal{T}_{h}$. These are described in [17] and [15], but for the sake of completeness, let us recall their definition. A vertex of $\mathcal{T}_{h}$ is singular if all the edges of $\mathcal{T}_{h}$ meeting at this vertex fall on two straight lines; otherwise, the vertex is nonsingular. Figure 1 


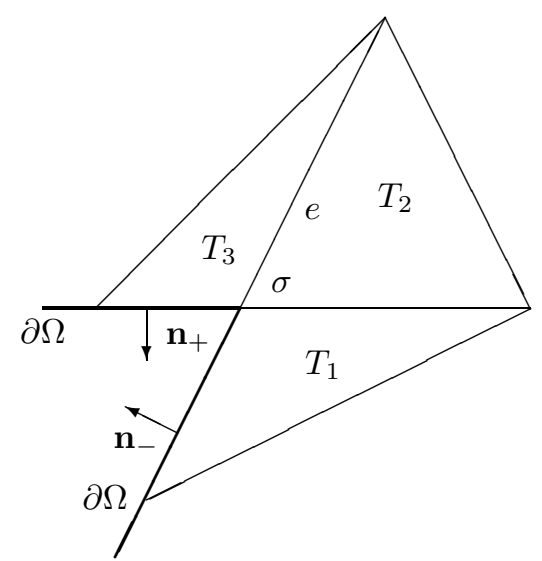

FiguRE 4. Boundary singular vertex where three triangles meet.

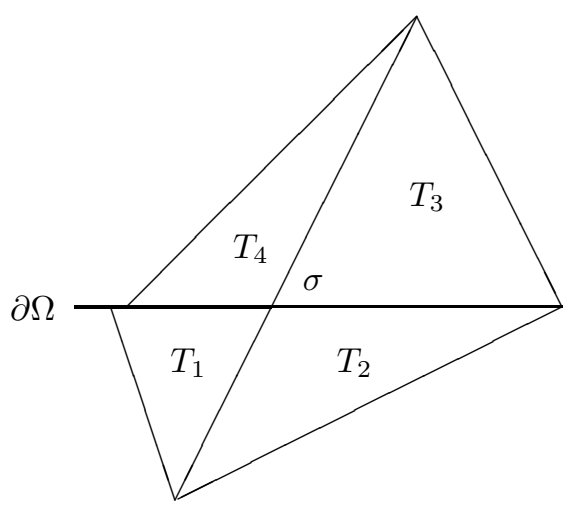

FigURE 5. Boundary singular vertex where four triangles meet: a slit domain.

depicts an internal singular vertex, i.e., one that belongs to $\mathcal{T}_{h} \cap \Omega$, and Figures $2[5$ illustrate the four types of boundary singular vertices, i.e., ones that belong to $\mathcal{T}_{h} \cap \partial \Omega$, the fourth type corresponding to a domain with a simple slit.

\section{3. $\mathcal{C}^{1}$ Finite ELEMENTS}

Let $\mathbb{P}_{r}^{d}$ be the space of polynomials of degree $r$ or less in $d$ variables. We consider a finite-element space $S_{h}^{r}$ consisting of $\mathcal{C}^{1}$ piecewise polynomials

$$
S_{h}^{r}:=\left\{v \in \mathcal{C}^{1}(\bar{\Omega}):\left.v\right|_{T} \in \mathbb{P}_{r}^{2} \forall T \in \mathcal{T}_{h}\right\}
$$

and the corresponding space with "homogeneous" Dirichlet boundary conditions:

$$
S_{h, c}^{r}=\left\{v \in S_{h}^{r}:\left.v\right|_{\Gamma_{i}} \text { is constant, } 0 \leq i \leq J,\left.\frac{\partial v}{\partial n}\right|_{\partial \Omega}=0\right\} .
$$

We choose this definition, with $v$ constant on each connected component of $\partial \Omega$, in order to discretize the stream functions of velocities that vanish on $\partial \Omega$. When only the normal component of the velocities vanishes, then the stream functions are constant on each $\Gamma_{i}$, with no constraint on the normal derivative, and in this case we are only interested in the first boundary condition of (3.2). 


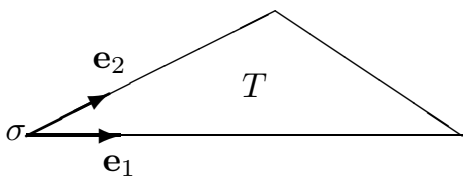

Figure 6. A triangle $T$ with edges $e_{1}$ and $e_{2}$ meeting at a vertex $\sigma$.

Before defining the nodal variables for $S_{h}^{r}$, let us recall the notions of secondorder edge-derivatives and second-order cross-derivatives. Let $T$ be a triangle, $\sigma$ one of its vertices, $\mathbf{e}_{1}$ and $\mathbf{e}_{2}$ the unit vectors along the edges of $T$ intersecting at $\sigma$, pointing away from $\sigma$, as in Figure [6] By the second $\mathbf{e}_{1}$-derivative of $f$ at $\sigma$, we mean

$$
\partial_{e_{1}} \partial_{e_{1}}\left(\left.f\right|_{T}\right)(\sigma),
$$

where $\partial_{e_{1}}$ denotes differentiation in the $\mathbf{e}_{1}$ direction. By the $T$-cross-derivative of $f$ at $\sigma$, we mean

$$
\partial_{e_{1}} \partial_{e_{2}}\left(\left.f\right|_{T}\right)(\sigma)
$$

For $r \geq 5$, a local, nodal basis for $S_{h}^{r}$ is given in [15. The following is a slight modification of the nodal representation in [15] for $S_{h}^{r}$, designed to preserve homogeneous boundary conditions. It consists of nodal variables of several types:

$$
\begin{aligned}
& \text { the value and gradient at the vertices of the triangulation; } \\
& \text { the second-order partial derivatives }(3.3) \text { in the direction of each } \\
& \text { edge, evaluated at each vertex, with the exception of one edge per } \\
& \text { nonsingular vertex (see below for the choice of edge omissions); } \\
& \text { one cross-derivative (3.4) at each vertex and two cross-derivatives } \\
& \text { at each nonsingular boundary vertex (see below for choices); } \\
& \text { the edge-normal derivative at } r-4 \text { distinct points in the interior } \\
& \text { of each edge; } \\
& \text { if } r>5 \text {, the value at } r-5 \text { distinct points in the interior of each } \\
& \text { edge; } \\
& \text { if } r>5 \text {, the value at dim } \mathbb{P}_{r-6}^{2} \text { distinct points in the interior of } \\
& \text { each triangle, chosen to determine uniquely an element of } \mathbb{P}_{r-6}^{2} \text {. }
\end{aligned}
$$

At an interior nonsingular vertex, the edge chosen for omitting a second-order derivative (3.3) in the direction of this edge, as described in (3.6), is such that its adjacent edges are not collinear. This is always possible, because the vertex would be singular if there were no such edges. At a boundary nonsingular vertex, the omitted edge is chosen to be an interior edge such that its adjacent edges are not collinear. Again, this is always possible, because otherwise the vertex is singular.

As far as cross-derivatives (3.4) are concerned, at an interior vertex $\sigma$, any $T$ cross-derivative may be chosen for any $T$ having $\sigma$ as a vertex. To satisfy boundary 
conditions, we will assume that any cross-derivative at a boundary vertex $\sigma$ is chosen to be associated with a triangle $T$ having a boundary edge. For a nonsingular boundary vertex, there are two such cross-derivatives to be specified, and we thus choose cross-derivatives associated with both of the triangles meeting there which have a boundary edge. For a singular boundary vertex, any triangle having a boundary edge can be picked. The following lemma shows that the corresponding finite elements do not depend on these particular choices of edge and cross-derivative.

Lemma 3.1. For any function $f \in S_{h}^{r}$, the second-order partial derivatives 3.6) and (3.7) at a node $\sigma$ determine the second-order edge-derivatives of $f$ at $\sigma$, on all the edges of $\mathcal{T}_{h}$ meeting at $\sigma$, and the $T$-cross-derivatives of $f$ at $\sigma$, on all the triangles $T$ of $\mathcal{T}_{h}$ having the common vertex $\sigma$.

Proof. The proof is based on arguments used in [15], but we reproduce them here for the reader's convenience. First observe that, if $\mathbf{e}_{1}$ is the unit vector along the edge between two triangles $T_{1}$ and $T_{2}$, as in Figure [7] then any $f \in S_{h}^{r}$ satisfies

$$
\partial_{e_{1}} \partial_{e_{1}}\left(\left.f\right|_{T_{1}}\right)(\sigma)=\partial_{e_{1}} \partial_{e_{1}}\left(\left.f\right|_{T_{2}}\right)(\sigma) \text {. }
$$

Similarly, for any $f \in S_{h}^{r}$, since $\partial_{e_{1}} f$ is continuous and is a piecewise polynomial, then $\partial_{e_{2}} \partial_{e_{1}} f$ is continuous along $\mathbf{e}_{2}$. For the same reason, $\partial_{e_{1}} \partial_{e_{2}} f$ is continuous along $\mathbf{e}_{1}$. Since these two partial derivatives are equal in each triangle, this implies that, with the notation of Figure 7

$$
\partial_{e_{1}} \partial_{e_{2}}\left(\left.f\right|_{T_{1}}\right)(\sigma)=\partial_{e_{1}} \partial_{e_{2}}\left(\left.f\right|_{T_{2}}\right)(\sigma)=\partial_{e_{1}} \partial_{e_{2}}\left(\left.f\right|_{T_{3}}\right)(\sigma)
$$

Now, consider again the triangles $T_{2}$ and $T_{3}$ of Figure 7 . It is easy to check that

$$
\partial_{e_{3}}=-\frac{\sin \theta_{2}}{\sin \theta_{1}} \partial_{e_{1}}+\frac{\sin \left(\theta_{1}+\theta_{2}\right)}{\sin \theta_{1}} \partial_{e_{2}} .
$$

Therefore

$$
\begin{aligned}
\partial_{e_{2}} \partial_{e_{3}}\left(\left.f\right|_{T_{3}}\right)(\sigma)= & -\frac{\sin \theta_{2}}{\sin \theta_{1}} \partial_{e_{1}} \partial_{e_{2}}\left(\left.f\right|_{T_{2}}\right)(\sigma) \\
& +\frac{\sin \left(\theta_{1}+\theta_{2}\right)}{\sin \theta_{1}} \partial_{e_{2}} \partial_{e_{2}}\left(\left.f\right|_{T_{2}}\right)(\sigma)
\end{aligned}
$$

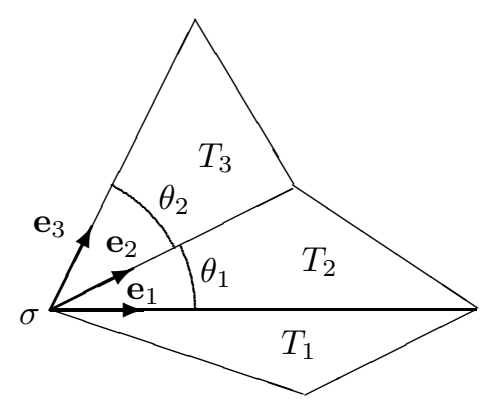

FiguRE 7. Three adjacent triangles with a common vertex $\sigma$. 
From this equation, we see that if we know the $T_{2}$-cross-derivative and the secondorder $\mathbf{e}_{2}$-edge-derivative, then we also know the $T_{3}$-cross-derivative. Thus, by progressing clockwise and counterclockwise from the known cross-derivative, and stopping at the edge where the second-order edge-derivative is omitted, or at a boundary edge, we can determine the cross-derivative in all the triangles of $\mathcal{T}_{h}$ having the common vertex $\sigma$.

Next, let $\sigma$ be a nonsingular vertex. Then we can assume that $\mathbf{e}_{1}$ and $\mathbf{e}_{3}$ are not collinear, we can invert (3.11), and we obtain

$$
\begin{aligned}
\partial_{e_{2}} \partial_{e_{2}}\left(\left.f\right|_{T_{2}}\right)(\sigma)= & \frac{\sin \theta_{1}}{\sin \left(\theta_{1}+\theta_{2}\right)} \partial_{e_{2}} \partial_{e_{3}}\left(\left.f\right|_{T_{3}}\right)(\sigma) \\
& +\frac{\sin \theta_{2}}{\sin \left(\theta_{1}+\theta_{2}\right)} \partial_{e_{1}} \partial_{e_{2}}\left(\left.f\right|_{T_{2}}\right)(\sigma) .
\end{aligned}
$$

Thus, if $\mathbf{e}_{2}$ is the edge where the second-order edge-derivative is omitted, and since we now know all the cross-derivatives, we can also determine this omitted secondorder derivative.

Finally, if $\sigma$ is a singular vertex, then $\theta_{1}+\theta_{2}=\pi$; hence $\sin \left(\theta_{1}+\theta_{2}\right)=0$ and $\sin \theta_{1}=\sin \theta_{2}$. Therefore (3.11) shows that the cross-derivatives on all the triangles having $\sigma$ as a common vertex are equal up to a sign. Since no secondorder edge-derivative is omitted at a singular vertex, this means that again all the second-order derivatives are determined at $\sigma$.

Clearly, formula (3.12) degenerates when a nonsingular vertex becomes singular. We shall see in the next section how a judicious choice of the omitted second-order edge-derivative prevents this degeneracy.

Also, one small point regarding the representation of the gradient in (3.5). For interior vertices, any choice is allowable, but for the boundary vertices, we impose some restrictions. At vertices on "smooth" parts of the boundary (i.e., where it is a straight segment), we choose the gradient to be represented by the normal and tangential derivatives. That is, the corresponding basis function has the normal (resp. tangential) derivative equal to one, with other nodal variables zero. At a "corner" point on the boundary, there are two tangential (and two normal) directions, so there would be ambiguity in the previous choice. In this case, we can choose the gradient to be represented either by the two tangential derivatives or by the two normal derivatives.

The above nodal variables coincide with those defined in [15] with only one exception. In the case of nonsingular boundary vertices, we omit one second-order edge derivative (3.3) and introduce instead two cross-derivatives (3.4), whereas in [15], no second-order edge-derivative is omitted, but there is only one crossderivative. Equations (3.11) and (3.12) show that our nodal variables are equivalent to the choice in [15]. Then the unisolvence of our nodal variables in $S_{h}^{r}$ follows easily from this equivalence and the unisolvence in $S_{h}^{r}$ of the nodal variables defined in [15]. Thus, we have the following theorem.

Theorem 3.2. For $r \geq 5$, there is a basis $\left\{\phi_{i}: i=1, \ldots, N\right\}$ of $S_{h}^{r}$ where each $\phi_{i}$ has the nodal value equal to one and all the other nodal values equal to zero.

Let us now summarize the nodal variables from a local perspective. In the interior of each triangle, there are $\operatorname{dim} \mathbb{P}_{r-6}^{d}$ distinct point-evaluation nodal variables as described in (3.10). In the interior of each edge, there are $r-4$ edge-normal 
derivative nodal variables (3.8) and $r-5$ point-evaluation nodal variables (3.9). At each vertex, the value and gradient (3.5) are nodal variables.

Suppose that the vertex is a nonsingular interior vertex. In addition, then there will be second-order directional derivatives (3.3) in the direction of each edge as nodal variables for all of the edges but one. If there are $k$ edges meeting at a vertex, this will add $k-1$ nodal variables. Finally, a cross-derivative (3.4) will also be a nodal variable. If there are $k$ edges meeting at a nonsingular interior vertex, this gives a total of $k+3$ nodal variables.

At a singular interior vertex, the number of nodal variables increases by one from what it would be if it were nonsingular. Since exactly $k=4$ edges meet at a singular interior vertex, this means that there are eight nodal variables at a singular interior vertex. The additional nodal variable results because there is a second-order directional derivative in the direction of each edge for all four edges (no omission). So the nodal variables consist of the point value and gradient (3.5), four second-order directional derivatives (3.6), and one cross-derivative (3.7).

At a nonsingular boundary vertex, the only difference between the corresponding nodal variables for a nonsingular interior vertex is the addition of a second cross-derivative (3.7). Moreover, these are chosen to correspond precisely to the two boundary triangles meeting at that vertex. If there are $k$ edges meeting at a nonsingular boundary vertex, then there is a total of $k+4$ nodal variables. At a singular boundary vertex, the nodal variables are in a sense identical to those for a singular interior vertex. That is, if $k$ edges meet at a singular boundary vertex (in which case $2 \leq k \leq 4$, or $k=5$ in the case of a simple slit), there are $k$ second-order directional derivatives in the direction of each edge for all $k$ edges (no omission), and only one cross-derivative (3.7). This gives a total of $k+4$ nodal variables at a singular boundary vertex, the same as for a nonsingular boundary vertex.

\section{4. $\mathcal{C}^{1}$ PIECEWISE POLYNOMIALS}

We begin with the standard definition of an interpolation operator on $W^{l, p}(\Omega)$, with $l \geq 4$ if $p=1$ and $l>2+2 / p$ otherwise. We denote the set of all interpolation nodal variables for the triangulation $\mathcal{T}_{h}$, as listed in Section [3, by $\mathcal{N}_{h}=\left\{v \rightarrow D_{i} v\left(a_{i}\right): i=1, \ldots, N\right\}$, and let $\left\{\phi_{i}: i=1, \ldots, N\right\}$ be the corresponding nodal basis of $S_{h}^{r}$. That is, for each $v \in S_{h}^{r}$,

$$
v=\sum_{i=1}^{N} D_{i} v\left(a_{i}\right) \phi_{i} .
$$

By Theorem 3.2 we know that such a basis exists. Each of the differential operators $D_{i}$ corresponds to one of the types of nodal variables listed above, and has order $\left|D_{i}\right|$ equal to zero (meaning just point evaluation), one or two. We will assume that all the nodal points $a_{i}$, when each element is mapped by an affine mapping to a reference element, are mapped to a fixed set of points, as would be the case for affine-equivalent elements in the Lagrange case. However, note that the edgenormal nodal variables do not get mapped to normal derivatives under such a mapping; rather, they are mapped to oblique derivatives in the general case [6].

Let $\Delta_{i}$ denote the union of the triangles of $\mathcal{T}_{h}$ containing $a_{i}$,

$$
h_{i}:=\max _{T \subset \Delta_{i}} h_{T}, \rho_{i}:=\min _{T \subset \Delta_{i}} \rho_{T} .
$$


In view of assumption (2.1), $\mathcal{T}_{h}$ is locally quasi-uniform, i.e.,

$$
\max _{1 \leq i \leq N} \frac{h_{i}}{\rho_{i}} \leq \gamma_{1}
$$

with a constant $\gamma_{1}$ independent of $h$.

Under the above assumptions on the mesh, bounds for the basis functions in (4.1) are standard, except for the fact that the global representation does not explicitly involve all the data required to define an interpolant locally. However, Lemma 3.1 and more precisely, the formulae (3.11) and (3.12), provide these data implicitly. As mentioned before, the only cause for concern is the apparent singularity in the representation (3.12) when a vertex is nearly singular. Let us now analyze what can happen in this case.

An interior vertex $\sigma$ can be "nearly singular" only when four vertices meet there. Let us number the four edges and angles cyclically, so that, e.g., $\theta_{0}$ is the same as $\theta_{4}$. We follow the notation of Figure 7 so that $\theta_{i}$ is the angle formed by the edges $e_{i}$ and $e_{i+1}$, and $e_{i+1}$ separates the angles $\theta_{i}$ and $\theta_{i+1}$. With this notation, let $\delta_{i}$ denote the denominator in (3.12), i.e.,

$$
\delta_{i}:=\sin \left(\theta_{i}+\theta_{i+1}\right),
$$

and let us choose the edge where the edge-derivative is omitted (without loss of generality, denote it by $e_{1}$ ) such that $\delta_{4}$ is the largest of all the $\delta_{i}$ 's (recall that $e_{1}$ separates $\theta_{4}$ and $\theta_{1}$, and $\delta_{4}=\sin \left(\theta_{4}+\theta_{1}\right)$ ). Note also that this choice implies necessarily one of the two following patterns:

$$
-\sin \left(\theta_{1}+\theta_{4}\right)=\sin \left(\theta_{2}+\theta_{3}\right)<\sin \left(\theta_{3}+\theta_{4}\right)<0<\sin \left(\theta_{1}+\theta_{2}\right)<\sin \left(\theta_{1}+\theta_{4}\right)
$$

or

$$
-\sin \left(\theta_{1}+\theta_{4}\right)=\sin \left(\theta_{2}+\theta_{3}\right)<\sin \left(\theta_{1}+\theta_{2}\right)<0<\sin \left(\theta_{3}+\theta_{4}\right)<\sin \left(\theta_{1}+\theta_{4}\right) .
$$

Otherwise said, the ratios $\delta_{i} / \delta_{4}$ are bounded, even as the vertex becomes singular.

Boundary vertices can be "nearly singular" only in a special case, which we shall legislate against by an assumption below. At an angular point on the boundary, the vertex there is always singular if only one triangle is there. If either two or more than three triangles meet at an angular point, degeneration toward singularity cannot occur. Only if three triangles meet at a nonconvex angular point can the vertex be "nearly singular," and we will assume that such vertices do not appear in the mesh.

Theorem 4.1. In addition to the above assumptions on the mesh, and choice of edge omission, suppose that at any angular point on the boundary, if exactly three triangles meet there, then the vertex is a singular vertex. Then for any real number $s \geq 0$ and for any number $p$ with $1 \leq p \leq \infty$,

$$
\left\|\phi_{i}\right\|_{W^{s, p}(\Omega)} \leq C h_{i}^{\left|D_{i}\right|-s+2 / p} .
$$

Proof. Let $\sigma$ be an interior vertex that is nearly singular, let $f_{i, j}$ denote the second derivative of $f$ with respect to the directions $\mathbf{e}_{i}$ and $\mathbf{e}_{j}$ at $\sigma$, and let $s_{i}=\sin \theta_{i}$. As chosen above, let $\delta_{4}$ be the largest of the $\delta_{i}$ 's and let $f_{1,1}$ be the omitted second-order edge-derivative. One can easily see that the cross-derivatives $f_{i, i+1}$ are determined in a bounded way from the given data (which are $f_{2,2}, f_{3,3}, f_{4,4}$, and, e.g., $f_{1,2}$, in this notation). But the second-edge-derivatives require a closer look. 
Define $g_{i}=f_{i, i} \delta_{i-1} / s_{i} s_{i-1}$ and $\tilde{g}_{i}=f_{i, i+1} / s_{i}$. Restating (3.11) in these general terms and dividing by $s_{i}$ yields

$$
g_{i}=\tilde{g}_{i}+\tilde{g}_{i-1}
$$

Multiplying by $(-1)^{i}$ and summing yields

$$
\sum_{i=1}^{4}(-1)^{i} g_{i}=0
$$

Using the definition of $g_{i}$, this may be written as

$$
f_{1,1}=\frac{s_{4}}{s_{2}} \frac{\delta_{1}}{\delta_{4}} f_{2,2}-\frac{s_{4}}{s_{3}} \frac{s_{1}}{s_{2}} \frac{\delta_{2}}{\delta_{4}} f_{3,3}+\frac{s_{1}}{s_{3}} \frac{\delta_{3}}{\delta_{4}} f_{4,4} .
$$

The assumption of nondegeneracy of the mesh implies that the terms $s_{i}$ are all bounded below uniformly, and our assumption about the choice of edge omission guarantees that the ratios $\delta_{i} / \delta_{4}$ all stay bounded. Thus the data in the global interpolant implicitly defines in a bounded way the data required to define the interpolant locally. More precisely, the local data for each $\phi_{i}$ on each triangle is bounded independently of $h$, so that (4.4) follows by standard arguments.

Let us now define a representation that is equivalent to (4.1) for $v \in S_{h}^{r}$ but is defined for functions with less regularity. To this end, we represent point evaluations by appropriate integrals, adapting to derivatives the representation of [18. To perform this integration, for any node $a_{i}$, we choose either a triangle or an edge $\kappa_{i}$ according to the type of $D_{i}$ and the position of $a_{i}$ with respect to the boundary, as follows. If $a_{i}$ is an interior point of some triangle $T \in \mathcal{T}_{h}$, we let

$$
\kappa_{i}=T \text {. }
$$

For all such nodes, $\left|D_{i}\right|=0$. If $a_{i}$ is an interior point of some edge $T^{\prime}$ of a triangle $T$ and if $T^{\prime}$ belongs to $\partial \Omega$, we let

$$
\kappa_{i}=T^{\prime}
$$

if $T^{\prime}$ is an interior edge, we could choose either this edge or one of the triangles sharing this edge. This applies for nodal variables with $\left|D_{i}\right|=0$ or 1 . We can see that this choice of $\kappa_{i}$ is not unique.

For the rest of the $a_{i}$, which must be vertices, if $\left|D_{i}\right|=0$ or 1 , we may pick any edge $T^{\prime}$, such that $a_{i} \in \overline{T^{\prime}}$, subject to the restriction

$$
T^{\prime} \subset \partial \Omega \quad \text { if } a_{i} \in \partial \Omega,
$$

and we set $\kappa_{i}=T^{\prime}$. Moreover, as explained just after Lemma 3.1, if $\left|D_{i}\right|=1$ and $a_{i}$ is a node on a smooth part of the boundary, then the same $\kappa_{i}$ is chosen for both components of the gradient, whereas, if $a_{i}$ is a "corner" point, each edge meeting at $a_{i}$ is chosen for each component of the gradient. If $a_{i}$ is not on the boundary, we could also choose any triangle $T$ sharing this vertex and set $\kappa_{i}=T$. Again, the choice of $\kappa_{i}$ is not unique. The restriction $T^{\prime} \subset \partial \Omega$ in (4.10) for $a_{i} \in \partial \Omega$ is made for the purpose of preserving homogeneous boundary conditions. Similarly, if $a_{i}$ is a vertex of $\overline{T^{\prime}}$ and $D_{i}$ denotes differentiation of second order in the direction of the edge $T^{\prime}$, we choose $\kappa_{i}=T^{\prime}$ if $T^{\prime}$ lies on $\partial \Omega$. If $T^{\prime}$ is an interior segment, we could also choose for $\kappa_{i}$ any triangle $T$ sharing this vertex. For the remaining type of nodal variable, i.e., the cross-derivative, if $T^{\prime}$ and $T^{\prime \prime}$ are the two edges supporting the vectors $\mathbf{e}_{1}$ and $\mathbf{e}_{2}$, then we can let $\kappa_{i}$ be either edge, or the triangle $T$ having 
these two edges, if $a_{i}$ is not on the boundary of $\Omega$. If $a_{i} \in \partial \Omega$, then by our choice of cross-derivative in this case, one of the two edges must be on the boundary, and we choose $\kappa_{i}$ to be that edge.

With such a choice of $\kappa=\kappa_{i}$, let $d=\operatorname{dim} \kappa$; i.e., $d=1$ if $\kappa$ is a segment and $d=2$ if $\kappa$ is a triangle. Take any point $z$ in $\kappa$. By Riesz's Representation Theorem, there exists a unique function $\psi_{z}^{\kappa} \in \mathbb{P}_{r}^{d}$ such that

$$
\int_{\kappa} f \psi_{z}^{\kappa} b_{\kappa} d \mu(\kappa)=f(z) \quad \forall f \in \mathbb{P}_{r}^{d},
$$

where $d \mu(\kappa)$ denotes the Lebesgue measure on $\kappa$. The weight $b_{\kappa}$ is the image on $\kappa$ of a polynomial $\hat{b}_{\kappa}$ of degree $d+1$ that vanishes to first order on the boundary $\partial \hat{\kappa}$ of the reference simplex $\hat{\kappa}$. For example, if $d=1$ and $\hat{\kappa}$ is the unit interval $[0,1]$, we take $\hat{b}_{\kappa}=\hat{x}(1-\hat{x})$; if $d=2$ and $\hat{\kappa}$ is the unit triangle $\{(\hat{x}, \hat{y}): 0 \leq \hat{x}, \hat{y} \leq 1, \hat{x}+\hat{y} \leq 1\}$, we take $\hat{b}_{\kappa}=\hat{x} \hat{y}(1-\hat{x}-\hat{y})$. We shall see below that the weight $b_{\kappa}$ is introduced in order to integrate by parts in (4.11).

By passing to the reference simplex $\hat{\kappa}$, formula 4.11 becomes

$$
\int_{\hat{\kappa}} \hat{f} \widehat{\psi_{z}^{\kappa}} \hat{b}_{\kappa} J d \mu(\hat{\kappa})=\hat{f}(\hat{z})
$$

where $J$ is the (constant) Jacobian of the transformation that maps $\hat{\kappa}$ onto $\kappa$. As this transformation maps $\mathbb{P}_{r}^{d}$ onto itself, and the Riesz-representation function is unique, this means that

$$
\widehat{J \psi_{z}^{\kappa}}=\widehat{\psi}_{\hat{z}}
$$

where $\widehat{\psi}_{\hat{z}}$ is the corresponding Riesz-representation function on $\hat{\kappa}$ with respect to the bubble function $\hat{b}_{\kappa}$. (We drop the superscript $\hat{\kappa}$ on $\widehat{\psi}_{\hat{z}}$ since there is only one $\hat{\kappa}$ for each dimension, $d=1$ or 2.) Therefore, there exists a constant $\widehat{C}_{1}$, that depends only on $r, d, \hat{\kappa}$ and $\hat{b}_{\kappa}$, such that

$$
\left\|\widehat{\psi}_{\hat{z}}\right\|_{L^{\infty}(\hat{\kappa})} \leq \hat{C}_{1} \quad \text { i.e., } \quad\left\|J \widehat{\psi_{z}^{\kappa}}\right\|_{L^{\infty}(\hat{\kappa})} \leq \widehat{C}_{1}
$$

But

$$
J \geq \widehat{C}_{2} h^{\operatorname{dim} \kappa},
$$

where $\widehat{C}_{2}$ depends on $\gamma_{0}$, but is independent of $\kappa$ and $h$; and

$$
\left\|b_{\kappa}\right\|_{L^{\infty}(\kappa)}=\left\|\hat{b}_{\kappa}\right\|_{L^{\infty}(\hat{\kappa})}=\widehat{C}_{3},
$$

where $\widehat{C}_{3}$ depends only on $\operatorname{dim} \kappa$. Therefore

$$
\left\|b_{\kappa} \psi_{z}^{\kappa}\right\|_{L^{\infty}(\kappa)}=\left\|\hat{b}_{\kappa} \widehat{\psi_{z}^{\kappa}}\right\|_{L^{\infty}(\hat{\kappa})} \leq \widehat{C}_{4} h^{-\operatorname{dim} \kappa} .
$$

Let $v$ belong to $S_{h}^{r}$ and let $D_{i} v\left(a_{i}\right)$ be any nodal variable in $\mathcal{N}_{h}$. By applying (4.11) with $f=D_{i} v$ and $z=a_{i}$, we may now write (4.1) as

$$
v=\sum_{i=1}^{N}\left(\int_{\kappa_{i}} D_{i} v \psi_{a_{i}}^{\kappa_{i}} b_{\kappa_{i}} d \mu\left(\kappa_{i}\right)\right) \phi_{i},
$$

where $\psi_{a_{i}}^{\kappa_{i}}$ denotes the particular Riesz-representation function corresponding to the node $a_{i}$ which is supported on $\kappa_{i}$.

To be able to interpolate functions with little regularity, while respecting boundary conditions, it remains to eliminate second derivatives in (4.13). When $\left|D_{i}\right|=0$ 
or 1 , we set $\widetilde{D}_{i}=D_{i}$ and $\widetilde{\psi}_{i}=\psi_{a_{i}}^{\kappa_{i}} b_{\kappa_{i}}$. When $\left|D_{i}\right|=2$, we integrate by parts on the corresponding $\kappa_{i}$ and obtain

$$
\int_{\kappa_{i}} D_{i} v \psi_{a_{i}}^{\kappa_{i}} b_{\kappa_{i}} d \mu\left(\kappa_{i}\right)=\int_{\kappa_{i}} \widetilde{D}_{i} v \widetilde{\psi}_{i} d \mu\left(\kappa_{i}\right),
$$

where $\left|\widetilde{D}_{i}\right|=1$ and

$$
\widetilde{\psi}_{i}=-\frac{\partial}{\partial e_{i}}\left(\psi_{a_{i}}^{\kappa_{i}} b_{\kappa_{i}}\right)
$$

where $\mathbf{e}_{i}$ denotes a unit direction (which would be the direction of $\kappa_{i}$ in the case of a one-dimensional $\kappa_{i}$ ). Thus we write (4.13) as follows:

$$
v=\sum_{i=1}^{N}\left(\int_{\kappa_{i}} \widetilde{D}_{i} v \widetilde{\psi}_{i} d \mu\left(\kappa_{i}\right)\right) \phi_{i}
$$

where $\left|\widetilde{D}_{i}\right|=0$ or 1 , and we derive from (4.12)

$$
\left\|\widetilde{\psi}_{i}\right\|_{L^{\infty}\left(\kappa_{i}\right)} \leq C h_{i}^{\left|\widetilde{D}_{i}\right|-\left|D_{i}\right|-\operatorname{dim} \kappa_{i}}
$$

Remark 4.2. Note that we integrate by parts at most once. Thus, we choose a bubble function $b_{\kappa_{i}}$ that vanishes to first order on $\partial \kappa_{i}$, to avoid boundary terms when we integrate by parts. If we needed to integrate by parts twice, then we could replace $b_{\kappa_{i}}$ by the bubble function $b_{\kappa_{i}}^{2}$. This would be useful if we wanted to construct an interpolant valid for functions only in $L^{1}$, but of course it would not make sense to talk of preserving boundary values for such functions. All the functions interpolated in the sequel will have at least an integrable derivative, so we do not consider this case further.

\section{5. $\mathcal{C}^{1}$ INTERPOLATION}

For a function to be interpolable by means of formula (4.15), its first derivative must have a trace in $L^{1}\left(\kappa_{i}\right)$, in order to take into account the case where $\left|\widetilde{D}_{i}\right|=1$ and $\kappa_{i}$ is a segment. From the trace theorem (cf. [16], [10] or [1]), we know that, for $l \geq 2$ when $p=1$ and $l>1+1 / p$ when $p>1$, we have $W^{l-1, p}(\Omega) \subset L^{1}\left(\kappa_{i}\right)$, for $1 \leq i \leq N$, i.e.,

$$
\forall f \in W^{l-1, p}(\Omega),\|f\|_{L^{1}\left(\kappa_{i}\right)} \leq C\|f\|_{W^{l-1, p}(\Omega)},
$$

where $C$ is a constant that depends only on $l, p, \Omega$ and $\kappa_{i}$. Therefore we assume that

$$
l \geq 2 \quad \text { if } \quad p=1 \quad \text { and } \quad l>1+1 / p \quad \text { otherwise. }
$$

In both cases, this holds when $l=2$. Also, to take into account boundary values of stream functions, we introduce the space

$$
W_{c}^{l, p}(\Omega):=\left\{f \in W^{l, p}(\Omega):\left.f\right|_{\Gamma_{i}} \text { is constant, } 0 \leq i \leq J,\left.\frac{\partial f}{\partial n}\right|_{\partial \Omega}=0\right\} .
$$

Then we define an interpolation operator,

$$
\Pi: W^{l, p}(\Omega) \rightarrow S_{h}^{r},
$$

by

$$
\forall v \in W^{l, p}(\Omega), \Pi v(x):=\sum_{i=1}^{N}\left(\int_{\kappa_{i}} \widetilde{D}_{i} v \widetilde{\psi}_{i} d \mu\left(\kappa_{i}\right)\right) \phi_{i}(x)
$$


Here $\Pi$ depends on the choices of $\kappa_{i}$ in (4.8)-(4.10), so that in fact we are defining a family of interpolation operators, but we use the notation $\Pi$ instead of $\Pi_{\left\{\kappa_{i}\right\}}$ for simplicity.

From (4.15), we conclude that $\Pi$ is a projection:

$$
\Pi v=v \quad \forall v \in S_{h}^{r} .
$$

Note that in particular, for all $i$,

$$
\forall v \in W^{l, p}(\Omega), D_{i} \Pi v\left(a_{i}\right)=\int_{\kappa_{i}} \widetilde{D}_{i} v \widetilde{\psi}_{i} d \mu\left(\kappa_{i}\right) .
$$

We have seen above that condition (5.2) guarantees that the nodal values (5.7) are well defined. Further, (5.2) guarantees the validity of the homogeneous boundary condition, in the following sense. If $v \in W^{l, p}(\Omega)$, with $l$ and $p$ satisfying (5.2), and $v$ is constant on a connected component of $\partial \Omega$, say $v=c$ on $\Gamma_{j}$, then $v-c$ vanishes on $\Gamma_{j}$. It follows from the choice of $\kappa_{i}$ in (4.9) and (4.10), and from (5.5) $-(5.7)$, that $\Pi(v-c)$ also vanishes on $\Gamma_{j}$. Hence

$$
\Pi v=c \text { on } \Gamma_{j} .
$$

If in addition $v$ satisfies $\frac{\partial v}{\partial n}=0$ on $\Gamma_{j}$, then by considering again $w=v-c$, for all multi-indices $\alpha$ with $|\alpha| \leq 1, w$ satisfies

$$
\left.D^{\alpha} w\right|_{\Gamma_{j}}=0 \text { in the sense of } L^{1}\left(\Gamma_{j}\right) .
$$

Again,

$$
D_{i} \Pi w\left(a_{i}\right)=0 \quad \forall a_{i} \in \Gamma_{j}
$$

with the exception of the second-order edge-derivatives for the interior edges. For a triangle on the boundary $\Gamma_{j}$ whose boundary vertices are nonsingular, it is evident that enough nodal variables vanish to guarantee that both the value and the normal derivative of $\Pi w$ vanish on the boundary edge. For a triangle with a boundary singular vertex, we have seen that the relevant cross-derivatives vanish independently of the choice made. Therefore $\Pi$ preserves the homogeneous boundary condition:

$$
\Pi: W_{c}^{l, p}(\Omega) \rightarrow S_{h, c}^{r} .
$$

We summarize the above results in the following theorem.

Theorem 5.1. Suppose that $r \geq 5$. Let $l$ and $p$ satisfy (5.2), and let $\mathcal{T}_{h}$ satisfy (2.1). Then the operator $\Pi$ defined in (5.5) is a projection from $W^{l, p}(\Omega)$ onto $S_{h}^{r}$, defined in (3.1), with the property that if $v=c$, a constant, on a connected component $\Gamma_{j}$ of $\partial \Omega$, then $\Pi v=c$ on $\Gamma_{j}$, and furthermore $\Pi$ maps $W_{c}^{l, p}(\Omega)$ defined in (5.3) onto the subspace $S_{h, c}^{r}$ defined in (3.2).

It is equally interesting to determine the image of the first two traces on $S_{h}^{r}$. By definition, $S_{h}^{r} \subset W^{2, \infty}(\Omega)$, so we must determine first what restrictions hold on the traces of such smooth functions onto a polygonal boundary. Let $v_{0}$ denote the trace of a function $v \in W^{2, \infty}(\Omega)$ and $v_{1}$ the trace of its normal derivative. As the gradient of $v$ at a vertex of $\partial \Omega$ must be uniquely defined, this condition may be expressed succinctly as

$$
\left(\begin{array}{l}
v_{0}^{\prime}(\sigma+) \\
v_{1}(\sigma+)
\end{array}\right)=\mathcal{R}(\theta)\left(\begin{array}{l}
v_{0}^{\prime}(\sigma-) \\
v_{1}(\sigma-)
\end{array}\right)
$$


where $v^{\prime}$ denotes the derivative $\frac{d v}{d \sigma}$ in the direction of the boundary, $\theta$ is the angular change in direction of the tangent at the vertex $\sigma$, and $\mathcal{R}(\theta)$ is the corresponding rotation matrix. In general, $f(\sigma \pm)$ denotes the evaluation of $f$ at $\sigma$ with limits taken from the two different sides of the corresponding edge. Similarly, $[f]_{\sigma}$ denotes the jump (or difference) of these two quantities:

$$
[f]_{\sigma}:=f(\sigma+)-f(\sigma-)
$$

Let $\mathcal{E}_{h}$ denote the subdivision of the boundary into segments that is induced by $\mathcal{T}_{h}$. Define $\widetilde{B}_{h}$ to be the following space of piecewise polynomials on the boundary of $\Omega$ :

$$
\widetilde{B}_{h}:=\left\{\left(v_{0}, v_{1}\right): v_{0} \in \mathcal{C}^{0}(\partial \Omega), v_{i} \in \mathcal{C}^{1-i}(\partial \Omega),\left.v_{i}\right|_{T^{\prime}} \in \mathbb{P}_{r-i}^{1} \forall T^{\prime} \in \mathcal{E}_{h}, i=0,1\right\} .
$$

By the notation $v_{0} \in \mathcal{C}^{1}(\partial \Omega)$ (respectively, $v_{1} \in \mathcal{C}^{0}(\partial \Omega)$ ), we mean that in the interior of any straight line segment $I$ of $\partial \Omega,\left.v_{0}\right|_{I}$ must be in $\mathcal{C}^{1}(I)$ (respectively, $\left.v_{1}\right|_{I}$ must be in $\left.\mathcal{C}^{0}(I)\right)$. Define the trace mapping

$$
\tau v:=\left(\left.v\right|_{\partial \Omega},\left.\frac{\partial v}{\partial n}\right|_{\partial \Omega}\right)
$$

Clearly, $\tau S_{h}^{r} \subset \widetilde{B}_{h}$, but the converse is not true. In addition to (5.10), $\tau S_{h}^{r}$ must satisfy one further condition related to boundary singular vertices in the mesh. To illustrate this condition, suppose first that the boundary lies on the $x$-axis and that there is an edge lying on the $y$-axis that meets the boundary, and that this is the only edge in $\mathcal{T}_{h}$ that intersects the origin. That is, precisely two boundary triangles meet at the origin. Suppose that $v \in S_{h}^{r}$. Since $\frac{\partial^{2} v}{\partial x \partial y}$ must have the same value at the origin coming from both sides of the vertical edge, then the normal derivative must be $\mathcal{C}^{1}$ there. In the general case, we have seen that the cross-derivative (3.4) of a function $v \in S_{h}^{r}$ is continuous at a boundary singular vertex where exactly two triangles meet. Again, we can assume that the two boundary edges lie on the $x$-axis. Let $e$ denote the interior edge meeting the $x$-axis at the boundary singular vertex $\sigma$, as in Figure 3 We can express the normal derivative in terms of the $x$-derivative and the directional derivative in the direction of $e$ :

$$
\frac{\partial}{\partial n}=\alpha \frac{\partial}{\partial e}+\beta \frac{\partial}{\partial x}
$$

where $\alpha \neq 0$. Differentiating (5.14) in the $x$-direction gives

$$
\frac{\partial}{\partial x} \frac{\partial}{\partial n}=\alpha \frac{\partial}{\partial x} \frac{\partial}{\partial e}+\beta \frac{\partial^{2}}{\partial x^{2}} .
$$

Then if $v \in S_{h}^{r}$ and $\left(v_{0}, v_{1}\right)=\tau v$, we have

$$
\left[v_{1}^{\prime}-\beta v_{0}^{\prime \prime}\right]_{\sigma}=0,
$$

and since the primes denote differentiation in the direction tangent to the boundary, this formula applies to the general case, where the boundary does not necessarily lie on the $x$-axis.

When three triangles meet at a boundary singular vertex $\sigma$, the constraint formula is similar. Let us assume that one of the boundary edges lies on the $x$-axis, and let $e$ denote the other edge of the triangle with the edge on the $x$-axis, as in 
Figure 4, We can write

$$
\begin{aligned}
& \frac{\partial}{\partial n_{+}}=\alpha_{+} \frac{\partial}{\partial e}+\beta_{+} \frac{\partial}{\partial x}, \\
& \frac{\partial}{\partial n_{-}}=\alpha_{-} \frac{\partial}{\partial x}+\beta_{-} \frac{\partial}{\partial e},
\end{aligned}
$$

where $n_{ \pm}$denote the two normal directions at $\sigma$, with the convention that on the "+" side of $\sigma$, the tangential direction is the $x$-axis, and on the "-" side of $\sigma$, the tangential direction is in the direction of $e$; and $\alpha_{ \pm} \neq 0$. A simple geometrical calculation shows that $\alpha_{+}=\alpha_{-}$and $\beta_{+}=\beta_{-}$. Therefore, differentiating the "+" expression in the $x$-direction and the "-" expression in the $e$-direction, applying this to $v \in S_{h}^{r}$ with $\left(v_{0}, v_{1}\right)=\tau v$ and using the continuity of the cross-derivatives (3.4), we recover (5.16) with the above convention of tangential direction. We leave it as an exercise to show that (5.16) also holds when there is only one triangle at a singular vertex (a triangle with two boundary edges, as in Figure 2). The next theorem summarizes these results.

Theorem 5.2. Suppose that $r \geq 5$. Define $B_{h}$ as the subset of $\widetilde{B}_{h}$ such that condition (5.10) holds at every vertex of $\partial \Omega$ and condition (5.16) holds at every boundary singular vertex $\sigma$ of the mesh $\mathcal{T}_{h}$. Then $\tau S_{h}^{r}=B_{h}$.

Proof. The inclusion $\tau S_{h}^{r} \subset B_{h}$ has just been proven. To see that the inclusion map is onto, we consider an edge $e$ in $\mathcal{E}_{h}$. If both vertices of $e$ are nonsingular, then there are sufficient nodal variables available to determine independently both $v=v_{0}$ and $\frac{\partial v}{\partial n}=v_{1}$, and the remaining nodal variables for $v$ can be set equal to zero. The only subtle point is to use (5.15) to write the tangential derivative of the normal derivative in terms of the cross-derivative (3.4) together with the secondorder derivative in the tangential direction. Although the latter is already specified in the definition of $v=v_{0}$, the cross-derivative can be adjusted appropriately to fit an arbitrary value of the tangential derivative of the normal derivative.

If one or both vertices of $e$ are singular, then we must use (5.16) together with (5.15) to see that it is sufficient to have only one cross-derivative as a degree of freedom at a singular vertex.

The last two theorems have the following corollary.

Corollary 5.3. We retain the assumptions of Theorems 5.1 and 5.2. Let $v \in$ $W^{l, p}(\Omega)$ be such that $\tau v \in B_{h}$. Then $\tau(\Pi v)=\tau v$.

Proof. By assumption, there exists $v_{h} \in S_{h}^{r}$ be such that $\tau v_{h}=\tau v$. Then $w=v-v_{h}$ belongs to $W_{0}^{m, p}(\Omega)$, where $m=\min (l, 2)$. Therefore $\Pi w \in S_{h, c}^{r}$, and the result follows from the fact that $\Pi$ is a projection.

\section{Boundary interpolation}

In this section, we construct and briefly discuss a boundary interpolation operator, with values in $B_{h}$, defined for traces of the form $\left(0, \frac{\partial v}{\partial n}\right)$ with $v \in W^{l, p}(\Omega), l$ and $p$ satisfying (5.2). More precisely, we are given $v_{0}=0$ and $v_{1}=\frac{\partial v}{\partial n}$, we know that $v$ exists, but the expression of $v$ is not known. This will be applied in the last section for the approximation of Navier-Stokes equations. 
It is instructive to consider the restriction on a given triangle $T$, lying on the boundary, of the representation (4.15). It can be written

$$
\begin{aligned}
\left.v\right|_{T}= & \sum_{j=1}^{\operatorname{dim} \mathbb{P}_{r}^{2}-4}\left(\int_{\kappa_{i_{j}}} \widetilde{D}_{i_{j}} v \widetilde{\psi}_{i_{j}} d \mu\left(\kappa_{i_{j}}\right)\right) \phi_{i_{j}} \\
& +\left(\sum_{j=1}^{X_{T}} c_{j}^{X} \int_{\kappa_{i_{j}^{X}}} \widetilde{D}_{i_{j}^{X}} v \widetilde{\psi}_{i_{j}^{X}} d \mu\left(\kappa_{i_{j}^{X}}\right)\right) \phi_{T}^{X} \\
& +\left(\sum_{j=1}^{U_{T}} c_{j}^{U} \int_{\kappa_{i_{j}^{U}}} \widetilde{D}_{i_{j}^{U}} v \widetilde{\psi}_{i_{j}^{U}} d \mu\left(\kappa_{i_{j}^{U}}\right)\right) \phi_{T}^{U} \\
& +\left(\sum_{j=1}^{L_{T}} c_{j}^{L} \int_{\kappa_{i_{j}^{L}}} \widetilde{D}_{i_{j}^{L}} v \widetilde{\psi}_{i_{j}^{L}} d \mu\left(\kappa_{i_{j}^{L}}\right)\right) \phi_{T}^{L} \\
& +\left(\sum_{j=1}^{R_{T}} c_{j}^{R} \int_{\kappa_{i_{j}^{L}}} \widetilde{D}_{i_{j}^{R}} v \widetilde{\psi}_{i_{j}^{R}} d \mu\left(\kappa_{i_{j}^{R}}\right)\right) \phi_{T}^{R} .
\end{aligned}
$$

Here, the latter two sums correspond to the second edge-derivative terms pointing inside $\Omega$, as in (3.3) for the "left" and "right" boundary vertices of $T$. The point is that the derivatives (3.3) are required to represent a function locally, but they may not be included in the global list of nodal variables in (4.15), i.e., they may correspond to "omitted" nodes. If either of these are actual nodal variables, then $L_{T}$ (or $R_{T}$ ) is one, reducing the sum to a single term. However, in general, one must use Lemma 3.1 to determine the required value in terms of available nodal variables at these vertices.

The " $U$ " term just before them corresponds to one of the second edge-derivative terms (3.3) for the interior vertex of $T$. The other second edge-derivative term is included in the first sum, as at most one edge will be omitted at each vertex. If both second edge-derivative terms (3.3) are actual nodal variables, then $U_{T}$ is one, reducing the sum to a single term. This occurs when the omitted edge is in another triangle at this vertex.

The " $X$ " term just before that corresponds to (possibly) the cross-derivative term (3.4) for the interior vertex of $T$. Again, if this is a nodal variable, then $X_{T}$ is one, reducing the sum to a single term.

The remaining $\operatorname{dim} \mathbb{P}_{r}^{2}-4$ nodal variables appear in the initial sum. A significant point is that the cross-derivative terms (3.4) for the "left" and "right" boundary vertices of $T$ are included in this set of $\operatorname{dim} \mathbb{P}_{r}^{2}-4$ nodal variables. In the case of a nonsingular boundary vertex, this is obvious from the choice described for the nodal variables (3.7). But in the case of a singular boundary vertex, there is only one nodal variable. However, in this case, all of the cross-derivatives at a singular vertex are identical up to a sign, so any one of them acts as a proxy for all the others. Thus, we may view them as available for the local description despite the fact that they may be playing a dual role in another element.

While the representation (6.1) is quite complex for representing $\left.v\right|_{T}$, it simplifies substantially when we look at boundary values and normal derivatives. In 
particular, if we denote $T \cap \partial \Omega$ by $T^{\prime}$, then

$$
\left.v\right|_{T^{\prime}}=\left.\sum_{j=1}^{\operatorname{dim} \mathbb{P}_{r}^{2}-4}\left(\int_{\kappa_{i_{j}}} \widetilde{D}_{i_{j}} v \widetilde{\psi}_{i_{j}} d \mu\left(\kappa_{i_{j}}\right)\right) \phi_{i_{j}}\right|_{T^{\prime}}
$$

since $\phi_{T}^{X}, \phi_{T}^{U}, \phi_{T}^{L}$, and $\phi_{T}^{R}$ vanish to second order on $T^{\prime}$. Furthermore, all $\kappa_{i_{j}} \subset \partial \Omega$, because otherwise the basis functions $\phi_{i_{j}}$ vanish to second order on $T^{\prime}$. In particular, this includes all of the basis functions associated with the nodal variables (3.10) and all of the basis functions associated with the nodal variables (3.8) and (3.9) for the interior edges.

By renumbering the boundary nodal values if necessary, we may write

$$
\begin{aligned}
\left.v\right|_{T^{\prime}} & =\left.\sum_{j=1}^{r+1}\left(\int_{\kappa_{i_{j}}} \widetilde{D}_{i_{j}}\left(\left.v\right|_{\kappa_{i_{j}}}\right) \widetilde{\psi}_{i_{j}} d t\right) \phi_{i_{j}}\right|_{T^{\prime}} \\
& =\sum_{j=1}^{r+1}\left(\int_{\kappa_{i_{j}}} \widetilde{D}_{i_{j}}\left(\left.v\right|_{\kappa_{i_{j}}}\right) \widetilde{\psi}_{i_{j}} d t\right) \phi_{j}^{T^{\prime}}
\end{aligned}
$$

where $\widetilde{D}_{i_{j}}$ denotes only tangential derivatives, $d t$ denotes the Lebesgue measure on the segments $\kappa_{i_{j}}$, and $\left\{\phi_{j}^{T^{\prime}}: j=1, \ldots, r+1\right\}$ denotes a basis for $\mathbb{P}_{r}^{1}$ of Argyris type [6], namely involving the value and the first and second derivatives at the endpoints of $T^{\prime}$, and $r-5$ interior values, provided we choose the gradient at each "corner" point of $\partial \Omega$ to be represented in terms of the tangential derivatives. Unfortunately, it appears that such a choice will not lead to the normal derivative being represented in terms of normal-derivative data.

However, if instead we choose the gradient at each "corner" point of $\partial \Omega$ to be represented in terms of the normal derivatives, then we can obtain the following (again by renumbering the boundary nodal values if necessary). Differentiating the representation (6.1) normal to $T^{\prime}$, we find that

$$
\begin{aligned}
\left.\frac{\partial v}{\partial n}\right|_{T^{\prime}}= & \left.\sum_{j=1}^{2}\left(\int_{T^{\prime}}\left(\left.\frac{\partial v}{\partial e_{j}}\right|_{T^{\prime}}\right) \widetilde{\psi}_{i_{j}} d t\right) \frac{\partial \phi_{i_{j}}}{\partial n}\right|_{T^{\prime}} \\
& +\left.\sum_{j=3}^{r}\left(\int_{\kappa_{i_{j}}}\left(\left.\frac{\partial v}{\partial n}\right|_{\kappa_{i_{j}}}\right) \widetilde{\psi}_{i_{j}} d t\right) \frac{\partial \phi_{i_{j}}}{\partial n}\right|_{T^{\prime}},
\end{aligned}
$$

where $e_{1}$ and $e_{2}$ are the interior edges of $T$, and all $\kappa_{i_{j}} \subset \partial \Omega$. The first two terms $(j=1,2)$ correspond to cross-derivative nodes. The main point is to see that the nodal variables involving cross-derivatives can be resolved into cross-derivatives involving the normal and tangent:

$$
\frac{\partial}{\partial n}=\left(\alpha \frac{\partial}{\partial e}+\beta \frac{\partial}{\partial t}\right)
$$

where $e$ denotes an interior edge of $T$, and $\alpha \neq 0$. Since all of these basis functions $\phi_{i_{j}}$ vanish on $T^{\prime}$, we find that

$$
\left.\frac{\partial \phi_{i_{j}}}{\partial n}\right|_{T^{\prime}}=\left.\alpha_{j} \frac{\partial \phi_{i_{j}}}{\partial e_{j}}\right|_{T^{\prime}} \text { for } j=1,2
$$


Thus (6.4) becomes

$$
\begin{aligned}
\left.\frac{\partial v}{\partial n}\right|_{T^{\prime}}= & \left.\sum_{j=1}^{2}\left(\int_{T^{\prime}}\left(\left.\frac{\partial v}{\partial n}\right|_{T^{\prime}}-\left.\beta_{j} \frac{\partial v}{\partial t}\right|_{T^{\prime}}\right) \widetilde{\psi}_{i_{j}} d t\right) \frac{\partial \phi_{i_{j}}}{\partial e_{j}}\right|_{T^{\prime}} \\
& +\left.\sum_{j=3}^{r}\left(\int_{\kappa_{i_{j}}}\left(\left.\frac{\partial v}{\partial n}\right|_{\kappa_{i_{j}}}\right) \widetilde{\psi}_{i_{j}} d t\right) \frac{\partial \phi_{i_{j}}}{\partial n}\right|_{T^{\prime}} \\
= & \sum_{j=1}^{2}\left(\int_{T^{\prime}}\left(\left.\frac{\partial v}{\partial n}\right|_{T^{\prime}}-\left.\beta_{j} \frac{\partial v}{\partial t}\right|_{T^{\prime}}\right) \widetilde{\psi}_{i_{j}} d t\right) \tilde{\phi}_{j}^{T^{\prime}} \\
& +\sum_{j=3}^{r}\left(\int_{\kappa_{i_{j}}}\left(\left.\frac{\partial v}{\partial n}\right|_{\kappa_{i_{j}}}\right) \widetilde{\psi}_{i_{j}} d t\right) \tilde{\phi}_{j}^{T^{\prime}},
\end{aligned}
$$

where $\left\{\tilde{\phi}_{j}^{T^{\prime}}: j=1, \ldots, r\right\}$ denotes a basis for $\mathbb{P}_{r-1}^{1}$ of Hermite type [6], namely involving the value and first derivative at the endpoints of $T^{\prime}$ and $r-4$ interior values. In particular, $\left.\frac{\partial \phi_{i_{j}}}{\partial e_{j}}\right|_{T^{\prime}}$ for $i=1,2$ are the basis functions associated with the derivative nodes.

In general, it appears that specifying the normal derivative independently of the boundary values is incompatible with (5.16). However, if $v$ is constant on each connected component of $\partial \Omega$, we conclude from (6.6) that

$$
\left.\frac{\partial v}{\partial n}\right|_{T^{\prime}}=\sum_{j=1}^{r}\left(\int_{\kappa_{i_{j}}}\left(\left.\frac{\partial v}{\partial n}\right|_{\kappa_{i_{j}}}\right) \widetilde{\psi}_{i_{j}} d t\right) \tilde{\phi}_{j}^{T^{\prime}} .
$$

We now return to the construction of a global boundary interpolant for data of the form $\left(0, v_{1}\right)$. Considering that $v_{1}$ is a normal derivative, we use the following nodal values, for $r \geq 5$ :

- the value of $v_{1}$ at $r-4$ distinct points in the interior of each boundary segment,

- the value of $v_{1}$ at each boundary vertex,

- the derivative of $v_{1}$ along the boundary on both sides of each nonsingular boundary vertex, and on one side only in the case of a singular boundary vertex (because it determines the derivative of $v_{1}$ along the boundary, on the other side of this boundary vertex).

It is worth noting that the general ambiguity of the normal derivative at a "corner" point of $\partial \Omega$ plays no role here, since the assumption $v_{0} \equiv 0$ implies that the gradient is zero at such points.

Let $N_{b}$ denote the number of these nodal values. With the notation of the preceding section, every function $\left(0, v_{1}\right) \in B_{h}$ can be expressed with

$$
v_{1}(x(t))=\sum_{i=1}^{N_{b}}\left(\int_{\kappa_{i}} v_{1}(y(t)) \widetilde{\psi}_{i}(y(t)) d t\right) \phi_{i}(x(t)) .
$$

Therefore, we define a boundary interpolation operator $\Pi_{b}$ for all $v \in W^{l, p}(\Omega)$ with $\tau v=\left(0, v_{1}\right)$ :

$$
\Pi_{b} v_{1}(x(t)):=\sum_{i=1}^{N_{b}}\left(\int_{\kappa_{i}} v_{1}(y(t)) \widetilde{\psi}_{i}(y(t)) d t\right) \phi_{i}(x(t)),
$$


and to avoid a multiplicity of notation, we set

$$
\Pi_{b}\left(0, v_{1}\right):=\left(0, \Pi_{b} v_{1}\right) .
$$

Then for $\tau v=\left(0, v_{1}\right)$, (6.7) allows one to show that

$$
\tau \Pi v=\left(0, \Pi_{b} v_{1}\right) .
$$

\section{Stability AND INTERPOLATION ERROR}

From now on, we shall keep the assumptions of Theorem 4.1. We now consider the stability of the interpolator (5.5). To simplify, we first take $l=2$ and we consider $v$ in $W^{2, p}(\Omega)$, for an arbitrary number $p \geq 1$. Let $\kappa_{i}$ be defined as in Section 4, i.e., $\kappa_{i}$ is either a triangle $T$ or an edge $T^{\prime}$ of $T$. It follows from the trace theorem and a homogeneity argument [18] that, regardless of whether $\kappa_{i}$ is a triangle or an edge, there exists a constant $C$, independent of $i, h$ and $T$, such that

$$
\begin{gathered}
\|v\|_{L^{1}\left(\kappa_{i}\right)} \leq C \sum_{k=0}^{1} h_{T}^{k+\operatorname{dim} \kappa_{i}-2 / p}|v|_{W^{k, p}(T)} \quad \forall v \in W^{1, p}(T), \\
\|D v\|_{L^{1}\left(\kappa_{i}\right)} \leq C \sum_{k=1}^{2} h_{T}^{k-1+\operatorname{dim} \kappa_{i}-2 / p}|v|_{W^{k, p}(T)} \quad \forall v \in W^{2, p}(T) .
\end{gathered}
$$

This yields the following stability result.

Theorem 7.1. Suppose that $r \geq 5$. Let $p$ and $q$ be two numbers with $1 \leq p, q \leq \infty$, let $v \in W^{2, p}(\Omega)$, and let $m$ be any nonnegative integer. Let $\mathcal{T}_{h}$ satisfy (2.1) and let $T \in \mathcal{T}_{h}$. Then, there exists a constant $C$, independent of $h, v$ and $T$, such that

$$
\|\Pi v\|_{W^{m, q}(T)} \leq C \sum_{k=0}^{2} h_{T}^{k-m+\frac{2}{q}-\frac{2}{p}}|v|_{W^{k, p}\left(S_{T}\right)},
$$

where

$$
S_{T}=\text { interior }\left(\bigcup\left\{\overline{T_{i}}: \overline{T_{i}} \cap \bar{T} \neq \emptyset, \quad T_{i} \in \mathcal{T}_{h}\right\}\right),
$$

and $\Pi$ is defined in (5.5).

Proof. Number the nodal variables for $T$ so that they are the first $n_{1}$ nodal variables. It follows then from (5.5), 4.2), 4.4), (4.16), (7.1) and (7.2) that, for any $v \in$ $W^{2, p}(\Omega)$,

$$
\begin{aligned}
\|\Pi v\|_{W^{m, q}(T)} & \leq \sum_{i=1}^{n_{1}}\left|\int_{\kappa_{i}} \tilde{D}_{i} v \tilde{\psi}_{i} d \mu\left(\kappa_{i}\right)\right|\left\|\phi_{i}\right\|_{W^{m, q}(T)} \\
& \leq C \sum_{i=1}^{n_{1}} h_{T}^{\left|D_{i}\right|-m+\frac{2}{q}}\left|\int_{\kappa_{i}} \tilde{D}_{i} v \tilde{\psi}_{i} d \mu\left(\kappa_{i}\right)\right| \\
& \leq C \sum_{i=1}^{n_{1}} h_{T}^{\left|\tilde{D}_{i}\right|-\operatorname{dim} \kappa_{i}-m+\frac{2}{q}} \int_{\kappa_{i}}\left|\tilde{D}_{i} v\right| d \mu\left(\kappa_{i}\right) \\
& \leq C \sum_{k=0}^{2} h_{T}^{-m+\frac{2}{q}+k-\frac{2}{p}}|v|_{W^{k, p}\left(S_{T}\right)} .
\end{aligned}
$$

Here $C$ denotes possibly different constants that are independent of $h, T$ and $v$. 
Remark 7.2. In fact (7.4) is a simplification, because we only need to take for $S_{T}$ a suitable union of triangles containing the sets $\kappa_{i}$ that are used for defining the degrees of freedom of $\Pi v$. We may use (7.4) as long as $T$ is not in the vicinity of a slit; of course, in this case, $S_{T}$ is connected. But, if $T$ is near a slit (e.g., $T$ adjacent to a slit), since the degrees of freedom of $\Pi v$ are defined on either side of the slit independently of each other, we can always choose $S_{T}$ so that it is connected.

Now, let $r \geq 5$ be an integer, let $p$ be a number with $1 \leq p \leq \infty$, and, to begin with, let $l$ be an integer such that $2 \leq l \leq r+1$. For $v \in W^{l, p}(\Omega)$, we propose to estimate $\|v-\Pi v\|_{S}$ for various Sobolev norms $\|\cdot\|_{S}$. For any $T \in \mathcal{T}_{h}$, any polynomial $q \in \mathbb{P}_{r}^{2}$, and any integer $m$ with $0 \leq m \leq l$, we have by (5.6) and Theorem 7.1 that

$$
\begin{aligned}
|v-\Pi v|_{W^{m, p}(T)} & \leq|v-q|_{W^{m, p}(T)}+|\Pi(v-q)|_{W^{m, p}(T)} \\
& \leq|v-q|_{W^{m, p}(T)}+C \sum_{k=0}^{2} h_{T}^{k-m}|v-q|_{W^{k, p}\left(S_{T}\right)} .
\end{aligned}
$$

Hence, we must estimate the infimum of $|v-q|_{W^{k, p}\left(S_{T}\right)}$ over all $q$ in $\mathbb{P}_{r}^{2}$. To this end, observe that we can write $S_{T}$ as follows:

$$
S_{T}=\operatorname{interior}\left(\bigcup_{1 \leq j \leq \lambda_{T}} \overline{A_{j}}\right)
$$

where $A_{j}$ is the interior of the closure of the union of pairs of triangles in $S_{T}$ that share a common edge. The regularity of $\mathcal{T}_{h}$ implies on one hand that the number $\lambda_{T}$ of such domains $A_{j}$ is bounded by a fixed constant $\lambda_{0}$, that is independent of $T$ and $h$. On the other hand, it implies that each domain $A_{j}$ is star-shaped with respect to a ball $B_{j}$ of radius $\rho$ times the diameter of $S_{T}$, where $\rho$ depends only on $\gamma_{0}$. In addition, the regularity of $\partial \Omega$ implies that $S_{T}$ is connected. Let $q_{j}$, for $1 \leq j \leq \lambda_{T}$, be any set of polynomials in $\mathbb{P}_{r}^{2}$. Then, arguing as in [8], Theorem 7.1, p. 458 , we derive for any $j$ such that $1 \leq j \leq \lambda_{T}$, and for all integers $k \geq 0$,

$$
\forall v \in W^{k, p}\left(S_{T}\right),\left|v-q_{j}\right|_{W^{k, p}\left(S_{T}\right)} \leq C \sum_{i=1}^{\lambda_{T}}\left|v-q_{i}\right|_{W^{k, p}\left(A_{i}\right)},
$$

where the constant $C$ depends only on $k, p, \gamma_{0}$ and $\lambda_{0}$. Let $j$ be an index such that $T \subset A_{j}$; with the choice $q=q_{j}$, we have from (7.6)

$$
|v-\Pi v|_{W^{m, p}(T)} \leq\left|v-q_{j}\right|_{W^{m, p}\left(A_{j}\right)}+C \sum_{k=0}^{2} h_{T}^{k-m} \sum_{i=1}^{\lambda_{T}}\left|v-q_{i}\right|_{W^{k, p}\left(A_{i}\right)} .
$$

For $1 \leq j \leq \lambda_{T}$, we choose

$$
q_{j}=Q_{j}^{l} v
$$

where $Q_{j}^{l} v$ is the Taylor polynomial of order $l$ (and hence of degree $l-1$ ) of $v$ averaged over $B_{j}$ (cf. [5], Definition 4.1.3). The above properties of $A_{j}$ allow us to apply the Bramble-Hilbert lemma in the form developed in [5], Lemma 4.3.8, and we obtain

$$
\left\|v-Q_{j}^{l} v\right\|_{W^{k, p}\left(A_{j}\right)} \leq C h_{T}^{l-k}|v|_{W^{l, p}\left(A_{j}\right)},
$$


where the constant $C$ is independent of $j, v, h$ and $T$. When substituting (7.8) into (7.7), this yields

$$
|v-\Pi v|_{W^{m, p}(T)} \leq C h_{T}^{l-m}|v|_{W^{l, p}\left(S_{T}\right)}
$$

with a constant $C$ that is independent of $v, h$ and $T$. When summing (7.9) over all elements $T \in \mathcal{T}_{h}$, we still obtain a bound with a constant independent of $h$. Indeed, the regularity of $\mathcal{T}_{h}$ implies that the number of occurrences of a given element $\tilde{T}$ in all the domains $S_{T}$ is bounded by a constant that depends on $\gamma_{0}$, but is independent of $\tilde{T}$ and $h$. Hence, we deduce our first main theorem.

Theorem 7.3. Suppose that $r \geq 5$. Let $p$ be a number such that $1 \leq p \leq \infty$ and let $l$ be an integer such that $2 \leq l \leq r+1$. Let $v \in W^{l, p}(\Omega)$ and let $\mathcal{T}_{h}$ satisfy (2.1). Then there exists a constant $C$, independent of $v$ and $h$, such that for any integer $m$ with $0 \leq m \leq l$

$$
\left(\sum_{T \in \mathcal{T}_{h}} h_{T}^{p(m-l)}|v-\Pi v|_{W^{m, p}(T)}^{p}\right)^{1 / p} \leq C\|v\|_{W^{l, p}(\Omega)},
$$

where $\Pi$ is defined in (5.5).

By letting $m=l$ and applying the triangle inequality, the following corollary is derived.

Corollary 7.4. Under the assumptions of Theorem 7.3, there exists a constant $C$, independent of $v$ and $h$, such that

$$
\left(\sum_{T \in \mathcal{T}_{h}}|\Pi v|_{W^{l, p}(T)}^{p}\right)^{1 / p} \leq C|v|_{W^{l, p}(\Omega)},
$$

where $\Pi$ is defined in (5.5).

Recalling that $h=\max _{T \in \mathcal{T}_{h}} h_{T}$, the statement of Theorem 7.3 can be simplified as follows:

Corollary 7.5. Under the assumptions of Theorem 7.3, there exists a constant $C$, independent of $v$ and $h$, such that

$$
\left(\sum_{T \in \mathcal{T}_{h}}|v-\Pi v|_{W^{m, p}(T)}^{p}\right)^{1 / p} \leq C h^{l-m}\|v\|_{W^{l, p}(\Omega)}, \quad 0 \leq m \leq l .
$$

For $m=0,1,2$, the statement of Theorem 7.3 can be extended to noninteger values of $l>2$ by interpolating between two consecutive integer values of $l$ in (7.12). This gives the second main theorem of this section.

Theorem 7.6. We keep the assumptions of Theorem 7.3, but we suppose that $l$ is a real number such that $2 \leq l \leq r+1$ and $m=0,1$ or 2 . Then there exists a constant $C$, independent of $v$ and $h$, such that

$$
|v-\Pi v|_{W^{m, p}(\Omega)} \leq C h^{l-m}\|v\|_{W^{l, p}(\Omega)} .
$$

Corollary 7.7. Under the assumptions of Theorem 7.3, there exists a constant $C$, independent of $h$, such that for all $\left(\psi_{0}, \psi_{1}\right) \in B_{h}$, there is a function $\psi \in S_{h}^{r}$ such that $\left.\psi\right|_{\partial \Omega}=\psi_{0},\left.\frac{\partial \psi}{\partial n}\right|_{\partial \Omega}=\psi_{1}$, and

$$
\|\psi\|_{W^{2, p}(\Omega)} \leq C\left(\left\|\psi_{0}\right\|_{W^{2-1 / p, p}(\partial \Omega)}+\left\|\psi_{1}\right\|_{W^{1-1 / p, p}(\partial \Omega)}\right) .
$$


Proof. Owing to (5.10), we can use the extension theorem of [10] to extend $\left(\psi_{0}, \psi_{1}\right)$ to $\tilde{\psi} \in W^{2, p}(\Omega)$ satisfying $\tau \tilde{\psi}=\left(\psi_{0}, \psi_{1}\right)$ and

$$
\|\tilde{\psi}\|_{W^{2, p}(\Omega)} \leq C\left(\left\|\psi_{0}\right\|_{W^{2-1 / p, p}(\partial \Omega)}+\left\|\psi_{1}\right\|_{W^{1-1 / p, p}(\partial \Omega)}\right) ;
$$

then we define $\psi=\Pi \tilde{\psi}$, and apply Corollary 7.4 with $l=2$.

Note that the results obtained so far easily extend to domains with simple slits. In fact, the critical condition is that the set $S_{T}$ in (7.4) be connected, and this can be arranged in such a case; see Remark 7.2 .

Remark 7.8. All results of this section are derived in the general case where some of the $\kappa_{i}$ may be segments. This is necessary for boundary nodal values, in order to preserve boundary conditions. But it is not necessary for defining nodal values that are not on the boundary. When $\kappa_{i}$ is a triangle, the stability estimate for the corresponding nodal value is derived as in (7.5), except that it holds for functions in $W^{1,1}\left(\kappa_{i}\right)$. This choice of $\kappa_{i}$ will be used in the last section for all interior nodal values.

\section{DiVERGENCE-FREE INTERPOLATION}

One application of $\mathcal{C}^{1}$ interpolation is the interpolation of vector functions satisfying divergence constraints [17]. Consider the space of vector functions

$$
W_{h}:=\left\{\mathbf{v} \in \mathcal{C}^{0}(\bar{\Omega})^{2}:\left.v_{i}\right|_{T} \in \mathbb{P}_{r-1}^{2} \forall T \in \mathcal{T}_{h}, i=1,2\right\} .
$$

Further, define

$$
Z_{h}:=\left\{\mathbf{v} \in W_{h}: \operatorname{div} \mathbf{v}=0, \int_{\Gamma_{j}} \mathbf{v} \cdot \mathbf{n} d \sigma=0,0 \leq j \leq J\right\} .
$$

Note that if $J=0$, then $\partial \Omega$ is connected and the integral boundary condition in (8.2) is implied by the zero divergence in $\Omega$. For $r \geq 5$, we have according to [17]

$$
\operatorname{curl} S_{h}^{r}=Z_{h}
$$

where $\operatorname{curl} v:=\left(\frac{\partial v}{\partial y},-\frac{\partial v}{\partial x}\right)$.

Equation (8.3) and Theorem 5.2 give the trace space of the functions of $Z_{h}$. Indeed, let $\mathbf{g}$ be the trace of a function $\mathbf{v} \in Z_{h}$. Then $\mathbf{v}=\operatorname{curl} \phi$ for some $\phi \in S_{h}^{r}$. Therefore, it follows from Theorem 5.2 that

$$
\left.\phi\right|_{\partial \Omega}=v_{0},\left.\frac{\partial \phi}{\partial n}\right|_{\partial \Omega}=v_{1}
$$

for some pair $\left(v_{0}, v_{1}\right)$ in $B_{h}$. Hence, denoting by $\mathbf{t}$ the unit tangent vector to $\partial \Omega$, oriented in the counter-clockwise direction, we have

$$
\begin{gathered}
\mathbf{g} \cdot \mathbf{n}=\mathbf{v} \cdot \mathbf{n}=(\operatorname{curl} \phi) \cdot \mathbf{n}=\frac{\partial \phi}{\partial \sigma}=v_{0}^{\prime}, \\
\mathbf{g} \cdot \mathbf{t}=\mathbf{v} \cdot \mathbf{t}=(\operatorname{curl} \phi) \cdot \mathbf{t}=-\frac{\partial \phi}{\partial n}=-v_{1} .
\end{gathered}
$$

Thus $\mathbf{g}$ has the following expression:

$$
\mathbf{g}=v_{0}^{\prime} \mathbf{n}-v_{1} \mathbf{t} .
$$


This suggests defining the trace space of functions of $Z_{h}$ as follows. First, we define the following space of piecewise polynomials on the boundary:

$$
\tilde{G}_{h}:=\left\{\mathbf{v} \in \mathcal{C}^{0}(\partial \Omega)^{2}:\left.v_{i}\right|_{T^{\prime}} \in \mathbb{P}_{r-1}^{1} \forall T^{\prime} \in \mathcal{E}_{h}, i=1,2\right\},
$$

where, as previously, $\mathcal{E}_{h}$ denotes the subdivision of the boundary into segments that is induced by $\mathcal{T}_{h}$. Then we set

$$
G_{h}:=\left\{\mathbf{g} \in \tilde{G}_{h}: \mathbf{g}=v_{0}^{\prime} \mathbf{n}-v_{1} \mathbf{t},\left(v_{0}, v_{1}\right) \in B_{h}\right\} .
$$

The next theorem summarizes this result.

Theorem 8.1. Suppose that $r \geq 5$. Let $\gamma Z_{h}$ denote the space of traces of functions of $Z_{h}$. Then $\gamma Z_{h}=G_{h}$, where $G_{h}$ is defined by (8.6).

To illustrate the constraints on $G_{h}$ given by this theorem, consider flow in a box $[0,1] \times[0,1]$ with inflow boundary conditions $\mathbf{g}(0, y)=(f(y), 0)$ on the left-hand side of the box. It is uncommon for mesh generators to have triangles with two edges on the boundary, but it is not uncommon to find a boundary singular vertex, e.g., at a point $(0, y)$ with $0<y<1$, where exactly two triangles meet. If the "other" edge (between these two triangles) is not perpendicular to the $y$-axis, then (8.6) and (5.16) imply that $f^{\prime}$ should be continuous at $y$.

Remark 8.2. More generally, if $v_{0}=0$ (as will be the case in the next two sections), then $\mathbf{g}=-v_{1} \mathbf{t}$. As $\mathbf{g} \in \mathcal{C}^{0}(\partial \Omega)^{2}$, then $v_{1}$ must vanish at the corners of $\partial \Omega$, so that necessarily (5.10) is satisfied. It remains to examine the boundary singular points. At a boundary singular vertex $\sigma$ where two triangles meet, as in the above example and as in Figure 3, formula (5.16) implies that $v_{1}^{\prime}$ is continuous at $\sigma$. At a boundary singular vertex $\sigma$ where either one or three triangles meet, formula (5.16) implies that $v_{1}^{\prime}(\sigma+)=-v_{1}^{\prime}(\sigma-)$, considering the convention used for the tangent direction on either side of $\sigma$.

\section{Nonhomogeneous Navier-Stokes equations}

Let us consider the steady nonhomogeneous Navier-Stokes equations in two dimensions. As usual, we introduce the spaces

$$
V=\left\{\mathbf{v} \in H_{0}^{1}(\Omega)^{2}: \operatorname{div} \mathbf{v}=0\right\}, L_{0}^{2}(\Omega)=\left\{q \in L^{2}(\Omega): \int_{\Omega} q(x) d x=0\right\} .
$$

To simplify the discussion, we take the exterior force $\mathbf{f}$ to be zero. Then, for a given boundary data $\mathbf{g}$, the Navier-Stokes equations have the variational form: Find $\mathbf{u} \in H^{1}(\Omega)^{2}$ and $p \in L_{0}^{2}(\Omega)$, such that

$$
\begin{aligned}
\forall \mathbf{v} \in H_{0}^{1}(\Omega)^{2} a(\mathbf{u}, \mathbf{v})+b(\mathbf{v}, p) & +R c(\mathbf{u} ; \mathbf{u}, \mathbf{v})=0, \\
b(\mathbf{u}, q) & =0, \\
\mathbf{u} & =\mathbf{g} \text { on } \partial \Omega,
\end{aligned}
$$

where $R$ is a given real constant,

$$
\begin{aligned}
a(\mathbf{u}, \mathbf{v}) & :=\int_{\Omega} \nabla \mathbf{u}: \nabla \mathbf{v} d x, \\
b(\mathbf{v}, q) & :=-\int_{\Omega}(\operatorname{div} \mathbf{v}) q d x, \\
c(\mathbf{v} ; \mathbf{u}, \mathbf{w}) & :=\int_{\Omega}(\mathbf{v} \cdot \nabla) \mathbf{u} \cdot \mathbf{w} d x .
\end{aligned}
$$


This is a nonlinear problem, so it is not clear that it will always have a solution. By using a famous result of Leray and Hopf (cf. [12], [11]), one can prove that (cf. [13], 9]), on a Lipschitz-continuous domain $\Omega$, this problem does indeed have a solution for arbitrary $R$ and data $\mathbf{g}$ in $H^{1 / 2}(\partial \Omega)^{2}$ satisfying

$$
\int_{\Gamma_{i}} \mathbf{g} \cdot \mathbf{n} d s=0,0 \leq i \leq J .
$$

This result is of limited interest from a physical point of view for at least two reasons. For large $R$, physical solutions would typically be time-dependent, but more importantly, since the $H^{1}$ norm of solutions constructed in this way increases exponentially with $R$, the size of the solution violates basic model assumptions.

Remark 9.1. The $H^{1}$ norm of solutions constructed via the Leray-Hopf lifting grow exponentially with $R$ because Leray and Hopf use a smooth truncating function near the boundary that takes the value 1 in a neighborhood of the boundary and whose support is contained in a region bounded above by $2 \exp (-O(R))$.

In this section, we propose to modify this construction in order to obtain solutions with $H^{1}$ norms of the order of $\sqrt{R}$, in the case where $\mathbf{g}$ is a tangential field, i.e.,

$$
\mathrm{g} \cdot \mathbf{n}=0
$$

and $\mathbf{g}$ belongs to $W^{1-1 / \alpha, \alpha}(\partial \Omega)^{2}$, for some $\alpha>2$, so that $\mathbf{g}$ belongs also to $L^{\infty}(\partial \Omega)^{2}$. This generalizes Lemma 3.1, p. 122 of [20, where $\partial \Omega$ is at least of class $\mathcal{C}^{3}$ and $\mathbf{g} \in \mathcal{C}^{3}(\partial \Omega)^{2}$.

Going back to problem (9.1), we know that there exists $\mathbf{u}_{\mathbf{g}}$ in $H^{1}(\Omega)^{2}$ such that (cf. [9])

$$
\mathbf{u}_{\mathbf{g}}=\mathbf{g} \text { on } \partial \Omega \text { and } \operatorname{div} \mathbf{u}_{\mathbf{g}}=0 \text { in } \Omega .
$$

Thus, $\mathbf{u}$ can be written in the form $\mathbf{u}=\mathbf{u}_{0}+\mathbf{u}_{\mathbf{g}}$, where $\mathbf{u}_{0} \in V$, and (9.1) is equivalent to: Find $\mathbf{u}_{0} \in V$ such that

$$
\begin{aligned}
\forall \mathbf{v} \in V, a\left(\mathbf{u}_{0}, \mathbf{v}\right) & +R c\left(\mathbf{u}_{0} ; \mathbf{u}_{0}, \mathbf{v}\right)+R c\left(\mathbf{u}_{0} ; \mathbf{u}_{\mathbf{g}}, \mathbf{v}\right) \\
& +R c\left(\mathbf{u}_{\mathbf{g}} ; \mathbf{u}_{0}, \mathbf{v}\right)=-a\left(\mathbf{u}_{\mathbf{g}}, \mathbf{v}\right)-R c\left(\mathbf{u}_{\mathbf{g}} ; \mathbf{u}_{\mathbf{g}}, \mathbf{v}\right) .
\end{aligned}
$$

Since $c\left(\mathbf{u}_{0} ; \mathbf{u}_{0}, \mathbf{u}_{0}\right)=c\left(\mathbf{u}_{\mathbf{g}} ; \mathbf{u}_{0}, \mathbf{u}_{0}\right)=0$, this problem has a solution provided the bilinear form $(\mathbf{w}, \mathbf{v}) \mapsto a(\mathbf{w}, \mathbf{v})+R c\left(\mathbf{w} ; \mathbf{u}_{\mathbf{g}}, \mathbf{v}\right)$ is elliptic on $V \times V$, for a suitable choice of $\mathbf{u}_{\mathrm{g}}$. In turn, this will imply existence of a solution $(\mathbf{u}, p)$ of (9.1). So, everything relies on the construction of an adequate lifting $\mathbf{u}_{\mathbf{g}}$.

First, we construct a divergence-free lifting of $\mathbf{g}$.

Lemma 9.2. Let $\Omega$ be Lipschitz continuous and let $\mathbf{g} \in W^{1-1 / \alpha, \alpha}(\partial \Omega)^{2}$ for some $\alpha>2$ be such that

$$
\int_{\partial \Omega} \mathbf{g} \cdot \mathbf{n} d s=0
$$

Then there exist $\mathbf{w} \in W^{1, \alpha}(\Omega)^{2}$ and a constant $T$ satisfying

$$
\begin{aligned}
& \mathbf{w}=\mathbf{g} \text { on } \partial \Omega, \operatorname{div} \mathbf{w}=0 \text { in } \Omega, \\
& \|\mathbf{w}\|_{W^{1, \alpha}(\Omega)} \leq T\|\mathbf{g}\|_{W^{1-1 / \alpha, \alpha}(\partial \Omega)} .
\end{aligned}
$$


We skip the proof because it is an easy application of the following inf-sup condition, valid in a Lipschitz-continuous domain (cf. [2]), for $\frac{1}{\alpha}+\frac{1}{\beta}=1,1<\alpha<$ $\infty$ :

$$
\inf _{q \in L_{0}^{\beta}(\Omega)} \sup _{\mathbf{v} \in W_{0}^{1, \alpha}(\Omega)^{2}} \frac{\int_{\Omega}(\operatorname{div} \mathbf{v}) q d x}{|\mathbf{v}|_{W^{1, \alpha}(\Omega)}\|q\|_{L^{\beta}(\Omega)}} \geq \mu>0 .
$$

Of course, for sufficiently small data, this lifting will make the bilinear form of (9.4) elliptic. So, the following analysis concerns the case where the data are such that (9.5) does not allow one to prove this ellipticity.

Now, owing to (9.3), there exists a function $\psi$ in $H^{1}(\Omega)$ such that

$$
\mathbf{w}=\operatorname{curl} \psi
$$

and as $\mathbf{w} \in W^{1, \alpha}(\Omega)^{2}$, this implies that necessarily $\psi \in W^{2, \alpha}(\Omega)$ and

$$
\|\psi\|_{W^{2, \alpha}(\Omega)} \leq C\|\mathbf{g}\|_{W^{1-1 / \alpha, \alpha}(\partial \Omega)} .
$$

Furthermore, since $\mathbf{w} \cdot \mathbf{n}=\mathbf{g} \cdot \mathbf{n}=0$, then $\psi$ is constant on $\partial \Omega$ :

$$
\left.\psi\right|_{\Gamma_{i}}=c_{i},\left|c_{i}\right| \leq C\|\psi\|_{H^{1}(\Omega)}, 0 \leq i \leq J
$$

where $\Gamma_{i}$ are the connected components of $\partial \Omega$. The idea of Leray and Hopf consists in truncating $\psi$ near the boundary in such a way that $c\left(\mathbf{v} ; \mathbf{u}_{\mathbf{g}}, \mathbf{v}\right)$ is small. Here, we follow this idea, but we shall use a different truncating function.

For $\varepsilon>0$ small enough, define

$$
\Omega_{\varepsilon}=\left\{x \in \Omega: d(x) \leq C_{\Omega} \varepsilon\right\},
$$

where $d(x)$ denotes the distance from $x$ to the boundary and $C_{\Omega}$ is a constant to be specified further on. Since $d$ is only Lipschitz continuous, we replace it by a regularized distance function $\Delta$, of class $\mathcal{C}^{2}$ (such as the one defined in [19, p.171), that satisfies

$$
\begin{gathered}
\forall x \in \Omega, c_{1} d(x) \leq \Delta(x) \leq c_{2} d(x), \\
\forall x \in \Omega,\left|\partial_{x}^{\alpha} \Delta(x)\right| \leq c_{3} d(x)^{1-|\alpha|}, \quad 1 \leq|\alpha| \leq 2,
\end{gathered}
$$

with constants that depend only on $\Omega$. Then we define the function $\lambda$ of one variable $t$ by

$$
\lambda(t)= \begin{cases}1 & \text { for } 0 \leq t \leq \frac{1}{2} \\ 2 t\left(t-\frac{3}{2}\right)^{2} & \text { for } \frac{1}{2} \leq t \leq \frac{3}{2} \\ 0 & \text { for } t \geq \frac{3}{2}\end{cases}
$$

and we scale $\lambda$ as follows:

$$
\forall x \in \Omega, \lambda_{\varepsilon}(x)=\lambda\left(\frac{\Delta(x)}{\varepsilon}\right) .
$$

As (9.9) implies that $\lambda_{\varepsilon}(x)$ vanishes for all $x \in \Omega$ such that $d(x) \geq \frac{3 \varepsilon}{2 c_{1}}$, we take

$$
C_{\Omega}=\frac{3}{2 c_{1}}
$$

and we assume that $\varepsilon>0$ is sufficiently small so that $\Omega_{\varepsilon}$ consists of mutually disjoint neighborhoods $\Omega_{\varepsilon, i}$ of each $\Gamma_{i}$ :

$$
\bar{\Omega}_{\varepsilon, i} \cap \bar{\Omega}_{\varepsilon, j}=\emptyset, \text { for } j \neq i .
$$


Therefore, $\operatorname{support}\left(\lambda_{\varepsilon}\right) \subset \Omega_{\varepsilon}$, and

$$
\left\|\lambda_{\varepsilon}\right\|_{L^{\infty}(\Omega)} \leq 1,\left\|\lambda_{\varepsilon}\right\|_{W^{1, \infty}(\Omega)} \leq \frac{C}{\varepsilon},\left\|\lambda_{\varepsilon}\right\|_{W^{2, \infty}(\Omega)} \leq \frac{C}{\varepsilon^{2}},
$$

where here and in the sequel, all constants $C$ are independent of $\varepsilon$. Then we take

$$
\mathbf{u}_{\mathrm{g}}=\operatorname{curl}\left(\lambda_{\varepsilon} \tilde{\psi}\right)
$$

where $\tilde{\psi}=\psi-c_{i}$ in $\Omega_{\varepsilon, i}, 0 \leq i \leq J$. Owing to (9.12), $\tilde{\psi}$ is a single-valued function, and (9.8) implies that $\tilde{\psi}$ vanishes on $\partial \Omega$. By construction, $\mathbf{u}_{\mathbf{g}} \in W^{1, \alpha}(\Omega)^{2}$ and $\operatorname{support}\left(\mathbf{u}_{\mathbf{g}}\right) \subset \Omega_{\varepsilon}$. Since $\tilde{\psi}$ vanishes on $\partial \Omega$, we can check that it satisfies the following Poincaré inequality, for any number $s$ with $1 \leq s \leq \infty$ :

$$
\|\tilde{\psi}\|_{L^{s}\left(\Omega_{\varepsilon}\right)} \leq C \varepsilon\|\nabla \psi\|_{L^{s}\left(\Omega_{\varepsilon}\right)} .
$$

Then we have the following lemmas that express the dependence of $\mathbf{u}_{\mathbf{g}}$ upon $\varepsilon$ and g.

Lemma 9.3. Let $\mathbf{g} \in W^{1-1 / \alpha, \alpha}(\partial \Omega)^{2}$ for some $\alpha>2$. For any number $s$ with $1 \leq s \leq \infty$, we have

$$
\left\|\mathbf{u}_{\mathbf{g}}\right\|_{L^{s}\left(\Omega_{\varepsilon}\right)} \leq C \varepsilon^{1 / s}\|\mathbf{g}\|_{W^{1-1 / \alpha, \alpha}(\partial \Omega)} .
$$

Proof. Expanding $\mathbf{u}_{\mathbf{g}}=\operatorname{curl}\left(\lambda_{\varepsilon} \tilde{\psi}\right)$ and using (9.13), (9.15) and the regularity of $\psi$, we derive

$$
\left\|\mathbf{u}_{\mathbf{g}}\right\|_{L^{s}\left(\Omega_{\varepsilon}\right)} \leq C\|\operatorname{curl} \psi\|_{L^{s}\left(\Omega_{\varepsilon}\right)} \leq C \varepsilon^{1 / s}\|\operatorname{curl} \psi\|_{L^{\infty}\left(\Omega_{\varepsilon}\right)}
$$

and (9.16) follows from (9.7).

Lemma 9.4. Let $\mathbf{g} \in W^{1-1 / \alpha, \alpha}(\partial \Omega)^{2}$ for some $\alpha>2$. We have

$$
\left\|\nabla \mathbf{u}_{\mathbf{g}}\right\|_{L^{2}\left(\Omega_{\varepsilon}\right)} \leq C \frac{1}{\varepsilon^{1 / 2}}\|\mathbf{g}\|_{W^{1-1 / \alpha, \alpha}(\partial \Omega)} .
$$

Proof. Expanding $\mathbf{u}_{\mathbf{g}}=\operatorname{curl}\left(\lambda_{\varepsilon} \tilde{\psi}\right)$ and applying (9.13) and (9.15), we derive

$$
\begin{aligned}
\left\|\nabla \mathbf{u}_{\mathbf{g}}\right\|_{L^{2}\left(\Omega_{\varepsilon}\right)} & \leq C\left(\frac{1}{\varepsilon^{2}}\|\tilde{\psi}\|_{L^{2}\left(\Omega_{\varepsilon}\right)}+\frac{1}{\varepsilon}|\psi|_{H^{1}\left(\Omega_{\varepsilon}\right)}+|\psi|_{H^{2}\left(\Omega_{\varepsilon}\right)}\right) \\
& \leq C\left(\frac{1}{\varepsilon}|\psi|_{H^{1}\left(\Omega_{\varepsilon}\right)}+|\psi|_{H^{2}\left(\Omega_{\varepsilon}\right)}\right) .
\end{aligned}
$$

Hence, the regularity properties of $\psi$ yield

$$
\left\|\nabla \mathbf{u}_{\mathbf{g}}\right\|_{L^{2}\left(\Omega_{\varepsilon}\right)} \leq C\left(\frac{1}{\varepsilon^{1 / 2}}|\psi|_{W^{1, \infty}\left(\Omega_{\varepsilon}\right)}+|\psi|_{H^{2}\left(\Omega_{\varepsilon}\right)}\right)
$$

and (9.17) follows from (9.7).

Lemma 9.5. Let $\mathbf{g} \in W^{1-1 / \alpha, \alpha}(\partial \Omega)^{2}$ for some $\alpha>2$ and let $|\cdot|$ denote the Euclidean norm. We have for $\mathbf{u}_{\mathbf{g}}$ defined by (9.14)

$$
\forall \mathbf{v} \in H_{0}^{1}(\Omega)^{2},\left\|\left|\mathbf{u}_{\mathbf{g}}\right||\mathbf{v}|\right\|_{L^{2}\left(\Omega_{\varepsilon}\right)} \leq C \varepsilon|\mathbf{v}|_{H^{1}\left(\Omega_{\varepsilon}\right)}\|\mathbf{g}\|_{W^{1-1 / \alpha, \alpha}(\partial \Omega)} .
$$

Proof. For $\mathbf{v} \in H_{0}^{1}(\Omega)^{2}$, we can write

$$
\left\|\left|\mathbf{u}_{\mathbf{g}}\right||\mathbf{v}|\right\|_{L^{2}\left(\Omega_{\varepsilon}\right)} \leq\left\|\mathbf{u}_{\mathbf{g}}\right\|_{L^{\infty}\left(\Omega_{\varepsilon}\right)}\|\mathbf{v}\|_{L^{2}\left(\Omega_{\varepsilon}\right)}
$$

Since $\mathbf{v} \in H_{0}^{1}(\Omega)^{2}$, we can apply (9.15) with $s=2$; then (9.16) with $s=\infty$ yields (9.18). 
Now, let $\mathbf{v} \in V$. As

$$
c\left(\mathbf{v} ; \mathbf{u}_{\mathbf{g}}, \mathbf{v}\right)=-c\left(\mathbf{v} ; \mathbf{v}, \mathbf{u}_{\mathbf{g}}\right),
$$

Lemma 9.5 implies that

$$
\forall \mathbf{v} \in H_{0}^{1}(\Omega)^{2},\left|c\left(\mathbf{v} ; \mathbf{u}_{\mathbf{g}}, \mathbf{v}\right)\right| \leq C \varepsilon\|\mathbf{g}\|_{W^{1-1 / \alpha, \alpha}(\partial \Omega)}|\mathbf{v}|_{H^{1}\left(\Omega_{\varepsilon}\right)}^{2}
$$

with a constant $C$ that is independent of $\varepsilon, \mathbf{v}$ and $\mathbf{g}$. The next theorem summarizes the results of this section.

Theorem 9.6. Let $\Omega$ be a Lipschitz-continuous domain of $\mathbb{R}^{2}$ and let $\mathbf{g} \in$ $W^{1-1 / \alpha, \alpha}(\partial \Omega)^{2}$ satisfy (9.3). Then the nonhomogeneous Navier-Stokes problem (9.1) has at least one solution $(\mathbf{u}, p) \in H^{1}(\Omega)^{2} \times L_{0}^{2}(\Omega)$, and there exists a constant $C$, independent of $R$, such that each solution satisfies

$$
|\mathbf{u}|_{H^{1}(\Omega)} \leq C \sqrt{R}\|\mathbf{g}\|_{W^{1-1 / \alpha, \alpha}(\partial \Omega)}^{3 / 2} .
$$

Proof. Let $\mathbf{u}$ be any solution of (9.1). For some $\varepsilon$ to be chosen further on, define the lifting $\mathbf{u}_{\mathbf{g}}$ by (9.14) and write $\mathbf{u}=\mathbf{u}_{0}+\mathbf{u}_{\mathrm{g}}$, where $\mathbf{u}_{0} \in V$ is a solution of (9.4). In view of (9.19), the choice $\mathbf{v}=\mathbf{u}_{0}$ in (9.4) yields

$$
\left(1-C R \varepsilon\|\mathbf{g}\|_{W^{1-1 / \alpha, \alpha}(\partial \Omega)}\right)\left|\mathbf{u}_{0}\right|_{H^{1}(\Omega)} \leq\left|\mathbf{u}_{\mathbf{g}}\right|_{H^{1}\left(\Omega_{\varepsilon}\right)}+R\left\|\mathbf{u}_{\mathbf{g}}\right\|_{L^{4}\left(\Omega_{\varepsilon}\right)}^{2} .
$$

Now, we take

$$
\varepsilon=\frac{1}{2 C R\|\mathbf{g}\|_{W^{1-1 / \alpha, \alpha}(\partial \Omega)}}
$$

and we can assume that $\varepsilon$ is small, since the analysis concerns large data. This gives

$$
|\mathbf{u}|_{H^{1}(\Omega)} \leq 3\left|\mathbf{u}_{\mathbf{g}}\right|_{H^{1}\left(\Omega_{\varepsilon}\right)}+2 R\left\|\mathbf{u}_{\mathbf{g}}\right\|_{L^{4}\left(\Omega_{\varepsilon}\right)}^{2} .
$$

Finally, (9.17) and (9.16) with $s=4$ imply

$$
|\mathbf{u}|_{H^{1}(\Omega)} \leq C\left(\frac{3}{\sqrt{\varepsilon}}\|\mathbf{g}\|_{W^{1-1 / \alpha, \alpha}(\partial \Omega)}+2 R \sqrt{\varepsilon}\|\mathbf{g}\|_{W^{1-1 / \alpha, \alpha}(\partial \Omega)}^{2}\right)
$$

and (9.20) follows by substituting (9.21) into this last inequality. Existence of a solution is a classical consequence of this a priori estimate.

\section{Discretizations of the Navier-Stokes equations}

In this section, we assume that $\Omega$ is a Lipschitz-continuous polygon, and we suppose that $\mathbf{g}$ is a given boundary data satisfying (9.3) and for some $\alpha>2$, $\mathrm{g} \in W^{1-1 / \alpha, \alpha}(\partial \Omega)^{2}$. Let $\mathcal{T}_{h}$ be a triangulation of $\bar{\Omega}$ satisfying (2.1) and let $\mathbf{g}_{h}$ be an approximation of $\mathbf{g}$ in $G_{h}$ such that

$$
\begin{gathered}
\mathbf{g}_{h} \cdot \mathbf{n}=0 \text { on } \partial \Omega \\
\left\|\mathbf{g}_{h}\right\|_{W^{1-1 / \alpha, \alpha}(\partial \Omega)} \leq C\|\mathbf{g}\|_{W^{1-1 / \alpha, \alpha}(\partial \Omega)} .
\end{gathered}
$$

For example, we might take $\mathbf{g}_{h}$ to be the restriction to $\partial \Omega$ of the interpolant of a divergence-zero extension of $\mathbf{g}$ to the whole domain. This is always possible, because $\mathbf{g}=-v_{1} \mathbf{t}$ and $\mathbf{g}_{h}$ can be constructed directly by interpolating $v_{1}$ on the boundary with the interpolation operator $\Pi_{b}$ defined by (6.9). Thus (6.10) implies that

$$
\mathrm{g}_{h}=\left.\operatorname{curl}(\Pi \varphi)\right|_{\partial \Omega}
$$


where $\operatorname{curl} \varphi$ is an extension of $\mathbf{g}$ in $\Omega$. Note that the expression of $\varphi$ is not needed for the interpolation of $\mathbf{g}$.

Let

$$
V_{h}=\left\{\mathbf{v}_{h} \in Z_{h}:\left.\mathbf{v}_{h}\right|_{\partial \Omega}=\mathbf{0}\right\},
$$

and consider the following discretization of the Navier-Stokes problem (9.1): Find $\mathbf{u}_{h} \in Z_{h}$ such that

$$
\begin{aligned}
\forall \mathbf{v}_{h} \in V_{h} a\left(\mathbf{u}_{h}, \mathbf{v}_{h}\right)+R c\left(\mathbf{u}_{h} ; \mathbf{u}_{h}, \mathbf{v}_{h}\right) & =0, \\
\mathbf{u}_{h} & =\mathbf{g}_{h} \text { on } \partial \Omega .
\end{aligned}
$$

Let $\Omega_{\varepsilon}$ be as in the preceding section, with the constant $C_{\Omega}$ defined by (9.11) and $\varepsilon$ defined by (9.21), i.e., $\varepsilon$ is of the order of $R^{-1}$. Let $\Omega_{\varepsilon, h}$ denote the union of all elements of $\mathcal{T}_{h}$ that intersect $\Omega_{\varepsilon}$ and let $h_{b}$ denote the maximum diameter of these elements. We propose to establish that, if $\Omega_{\varepsilon, h}$ consists of mutually disjoint neighborhoods of the connected components $\Gamma_{j}$ and if

$$
h_{b}<\frac{\varepsilon}{2 c_{2}},
$$

i.e., $h_{b}$ is also of the order of $R^{-1}$, then problem (10.4) has at least one solution. It seems reasonable that the boundary layer be scaled by $O\left(R^{-1}\right)$, although we do not claim that this is optimal.

As for the continuous problem, this relies on the construction of an adequate lifting. First, since $\mathbf{g}_{h} \in G_{h}$, Theorem 8.1] and Corollary 7.7] show that there exists $\psi_{h} \in S_{h, c}^{r}$ such that

$$
\mathbf{g}_{h}=\left.\operatorname{curl} \psi_{h}\right|_{\partial \Omega}
$$

as in the continous case, $\psi_{h}$ is constant on each $\Gamma_{i}:\left.\psi_{h}\right|_{\Gamma_{i}}=c_{i}$, for $0 \leq i \leq J$, and (7.14) with $p=\alpha$ implies that $\psi_{h} \in W^{2, \alpha}(\Omega)$ and

$$
\left\|\psi_{h}\right\|_{W^{2, \alpha}(\Omega)} \leq C\left\|\mathbf{g}_{h}\right\|_{W^{1-1 / \alpha, \alpha}(\partial \Omega)} .
$$

Let $\lambda_{\varepsilon}$ be the truncating function constructed in the previous section, and define

$$
\mathbf{u}_{h, \mathbf{g}}=\operatorname{curl}\left(\Pi\left(\lambda_{\varepsilon} \tilde{\psi}_{h}\right)\right),
$$

where $\tilde{\psi}_{h}=\psi_{h}-c_{i}$ in $\Omega_{\varepsilon, i}, 0 \leq i \leq J$, and all the interior nodal values $\Pi v\left(a_{i}\right)$ are defined by choosing triangles for $\kappa_{i}$. According to Remark 7.8, this choice is justified by the stability result of Lemma 10.2 below, which does not hold if we use segments for $\kappa_{i}$ in the interior nodal values.

Since (9.9) implies that $\lambda_{\varepsilon}(x)$ is identically one on all $x \in \Omega$ such that $d(x) \leq \frac{\varepsilon}{2 c_{2}}$ and $h_{b}$ satisfies (10.5), then

$$
\left.\lambda_{\varepsilon} \tilde{\psi}_{h}\right|_{T}=\left.\tilde{\psi}_{h}\right|_{T}
$$

on all triangles $T$ that intersect the boundary $\partial \Omega$. Thus, as $\tilde{\psi}_{h}$ belongs to $W^{2, p}(\Omega)$ for any $p$, Corollary 5.3 implies that

$$
\left.\mathbf{u}_{h, \mathbf{g}}\right|_{\partial \Omega}=\mathbf{g}_{h} .
$$

Remark 10.1. One may ask: why not use $\operatorname{curl}\left(\Pi\left(\lambda_{\varepsilon} \tilde{\psi}\right)\right)$ instead of (10.7)? Of course, the trace of $\operatorname{curl}\left(\Pi\left(\lambda_{\varepsilon} \tilde{\psi}\right)\right)$ is the discrete boundary data $\mathbf{g}_{h}$, but the reason for not using it is that if we define $\mathbf{u}_{h, \mathbf{g}}$ by $\operatorname{curl}\left(\Pi\left(\lambda_{\varepsilon} \tilde{\psi}\right)\right)$ then $\mathbf{u}_{h, \mathbf{g}}$ will not satisfy the analogue of (10.8), namely it will not be bounded by $C\left|\lambda_{\varepsilon} \tilde{\psi}\right|_{W^{1, s}(\Omega)}$. Indeed, this bound requires that $\Pi$ be stable in $W^{1, s}(T)$ on triangles $T$ adjacent to the 
boundary, and this stability does not hold. In contrast, (10.8) holds because $\tilde{\psi}_{h}$ belongs to a finite-dimensional space. This property is used in (10.9). Summing up, the choice (10.7) leads to a "super-approximation" result which is valid only because $\lambda_{\varepsilon}=1$ (identically) where the interpolant is not so stable, and the function being interpolated is already in the finite element space.

Note also that if $\lambda_{\varepsilon}=1$ in a neighborhood of the boundary sufficiently wide to contain two rows of triangles, then on the triangles adjacent to the boundary, $\Pi\left(\lambda_{\varepsilon} \tilde{\psi}_{h}\right)=\tilde{\psi}_{h}$. Indeed, all the degrees of freedom used by $\Pi$ in the triangles adjacent to the boundary involve only those values of $\tilde{\psi}_{h}$ where $\lambda_{\varepsilon}=1$. But this may not be the case if the neighborhood of the boundary where $\lambda_{\varepsilon}=1$ is not wide enough.

Lemma 10.2. Let $\mathcal{T}_{h}$ satisfy (2.1) and $h_{b}$ satisfy (10.5). For any number $s$ with $1 \leq s \leq \infty$, the lifting function $\mathbf{u}_{h, \mathbf{g}}$ defined by (10.7) satisfies

$$
\left\|\mathbf{u}_{h, \mathbf{g}}\right\|_{L^{s}(\Omega)} \leq C\left|\lambda_{\varepsilon} \tilde{\psi}_{h}\right|_{W^{1, s}(\Omega)}
$$

with a constant $C$ independent of $h$ and $\varepsilon$.

Proof. We have

$$
\left\|\mathbf{u}_{h, \mathbf{g}}\right\|_{L^{s}(\Omega)}=\left|\Pi\left(\lambda_{\varepsilon} \tilde{\psi}_{h}\right)\right|_{W^{1, s}(\Omega)} .
$$

Since the interior nodal values of $\Pi(v)$ are defined for $v \in W^{1,1}\left(\kappa_{i}\right)$ and $\kappa_{i}$ are triangles, it suffices to examine boundary nodal values. Thus, let $\kappa_{i}$ be an edge of a triangle $T$ that intersects $\partial \Omega$. We can always assume that $T$ is adjacent to the boundary. From the choice of $h_{b},(4.4),(4.15)$ and (4.16), we derive

$$
\begin{aligned}
\mid\left(\int_{\kappa_{i}} \tilde{D}_{i}\left(\lambda_{\varepsilon} \tilde{\psi}_{h}\right) \tilde{\psi}_{i} d \mu\left(\kappa_{i}\right)\right) & \left.\phi_{i}\right|_{W^{1, s}(\Omega)} \\
& =\left|\int_{\kappa_{i}}\left(\tilde{D}_{i} \tilde{\psi}_{h}\right) \tilde{\psi}_{i} d \mu\left(\kappa_{i}\right)\right|\left|\phi_{i}\right|_{W^{1, s}(\Omega)} \\
& \leq C h_{i}^{\left|\tilde{D}_{i}\right|-2+2 / s} \int_{\kappa_{i}}\left|\tilde{D}_{i} \tilde{\psi}_{h}\right| d \mu\left(\kappa_{i}\right) .
\end{aligned}
$$

If $\left|\tilde{D}_{i}\right|=0$, considering that $\tilde{\psi}_{h}$ vanishes on one side of $T$, and passing to the reference unit triangle $\hat{T}$, we obtain

$$
\int_{\kappa_{i}}\left|\tilde{\psi}_{h}\right| d \mu\left(\kappa_{i}\right) \leq C h_{T} \int_{\hat{T}}\left\|\nabla \hat{\psi}_{h}\right\| d \hat{y} \leq C h_{T}\left\|\nabla \hat{\psi}_{h}\right\|_{L^{s}(\hat{T})} \leq C h_{T}^{2-2 / s}\left|\psi_{h}\right|_{W^{1, s}(T)} .
$$

If $\left|\tilde{D}_{i}\right|=1$, we have instead

$$
\begin{aligned}
\int_{\kappa_{i}}\left|\tilde{D}_{i} \tilde{\psi}_{h}\right| d \mu\left(\kappa_{i}\right) & \leq C \int_{\hat{T}}\left\|\nabla \hat{\psi}_{h}\right\| d \hat{y} \\
& \leq C\left\|\nabla \hat{\psi}_{h}\right\|_{L^{s}(\hat{T})} \\
& \leq C h_{T}^{1-2 / s}\left|\psi_{h}\right|_{W^{1, s}(T)} .
\end{aligned}
$$

Here we have replaced the trace theorem by an equivalence of norms, valid in a finite-dimensional space. Hence, using again the fact that $\lambda_{\varepsilon}=1$ on $T$, we have in both cases

$$
\left|\left(\int_{\kappa_{i}} \tilde{D}_{i}\left(\lambda_{\varepsilon} \tilde{\psi}_{h}\right) \tilde{\psi}_{i} d \mu\left(\kappa_{i}\right)\right) \phi_{i}\right|_{W^{1, s}(\Omega)}=C\left|\lambda_{\varepsilon} \tilde{\psi}_{h}\right|_{W^{1, s}(T)} .
$$


Furthermore, it follows from (17.11) that

$$
\left\|\nabla \mathbf{u}_{h, \mathbf{g}}\right\|_{L^{2}(\Omega)} \leq C\left|\lambda_{\varepsilon} \tilde{\psi}_{h}\right|_{H^{2}(\Omega)} .
$$

Then in view of (9.13), (10.6) and (10.2), we derive the analogue of Theorem 9.6

Theorem 10.3. Let $\Omega$ be a Lipschitz-continuous polygon, let $\mathbf{g} \in W^{1-1 / \alpha, \alpha}(\partial \Omega)^{2}$ for some $\alpha>2$ satisfy (9.3) and let $\mathcal{T}_{h}$ satisfy (2.1). Let $\mathbf{g}_{h} \in G_{h}$ be an approximation of $\mathbf{g}$ satisfying (10.1) and (10.2). There exists a constant $C$ such that if $h_{b}<\frac{C}{R}$, then the nonhomogeneous discrete Navier-Stokes problem (10.4) has at least one solution $\mathbf{u}_{h}$ in $Z_{h}$, and each solution satisfies

$$
\left|\mathbf{u}_{h}\right|_{H^{1}(\Omega)} \leq C \sqrt{R}\|\mathbf{g}\|_{W^{1-1 / \alpha, \alpha}(\partial \Omega)}^{3 / 2}
$$

with another constant $C$ independent of $h$ and $R$.

A result of this type could be proved using a perturbation argument, based on picking $\mathbf{u}_{h}$ as a projection of $\mathbf{u}$, e.g., by solving a discrete Stokes equation. But then the $h$ would have to be a global measure of mesh size, instead of a local mesh size at the boundary, unless suitable local estimates for the projection were developed. Thus using the specialized interpolant introduced here leads to at least a simpler derivation of the estimate, and it may be a sharper estimate than would be possible by perturbation as well.

As mentioned at the beginning of this section, $\mathbf{g}_{h}$ can be constructed of the form (10.3), where $\operatorname{curl} \varphi$ is an extension of $\mathbf{g}$ in $\Omega$. Therefore, setting

$$
P_{h}(\mathbf{u})=\operatorname{curl}(\Pi \psi),
$$

where $\psi$ is the stream function of $\mathbf{u}$, Theorem 5.1 implies that

$$
P_{h}(\mathbf{u})=\mathbf{g}_{h} \text { on } \partial \Omega
$$

because $\operatorname{curl} \psi$ is necessarily an extension of $\mathbf{g}$ in $\Omega$, and thus $\varphi-\psi \in H_{c}^{2}(\Omega)$. Then a straightforward argument and Theorem 10.3 prove the following convergence result.

Theorem 10.4. In addition to the hypotheses of Theorem 10.3, suppose that $\mathbf{g}_{h}$ satisfies (10.3). Then, as $h$ tends to zero, each solution $\mathbf{u}_{h}$ of (10.4) converges strongly in $H^{1}(\Omega)^{2}$ to a solution $\mathbf{u}$ of (9.1).

Remark 10.5. With a slight loss of exponents, the results of this section can be extended to the case where $\mathbf{g}$ belongs only to $H^{1 / 2}(\partial \Omega)^{2}$. Thus, (9.16) is replaced by

$$
\left\|\mathbf{u}_{\mathbf{g}}\right\|_{L^{s}\left(\Omega_{\varepsilon}\right)} \leq C \varepsilon^{1 / s-1 / t}\|\mathbf{g}\|_{H^{1 / 2}(\partial \Omega)}, \text { for } 1 \leq s \leq t<\infty
$$

and (9.17) is replaced by

$$
\left\|\nabla \mathbf{u}_{\mathbf{g}}\right\|_{L^{2}\left(\Omega_{\varepsilon}\right)} \leq C \frac{1}{\varepsilon^{1 / 2+1 / t}}\|\mathbf{g}\|_{H^{1 / 2}(\partial \Omega)}, \text { for } 2 \leq t<\infty .
$$

As far as (9.18) is concerned, we assume that $\Omega$ is a Lipschitz polygon. Then, by a scaling argument and an application of Sobolev's inequality, we derive

$$
\forall v \in H_{0}^{1}(\Omega),\|v\|_{L^{s}\left(\Omega_{\varepsilon}\right)} \leq C \varepsilon^{2 / s}|v|_{H^{1}\left(\Omega_{\varepsilon}\right)}, \text { for } 2 \leq s<\infty .
$$

Thus, (9.18) is replaced by

$$
\forall \mathbf{v} \in H_{0}^{1}(\Omega)^{2},\left\|\left|\mathbf{u}_{\mathbf{g}}\right||\mathbf{v}|\right\|_{L^{2}\left(\Omega_{\varepsilon}\right)} \leq C \varepsilon^{1 / t}|\mathbf{v}|_{H^{1}\left(\Omega_{\varepsilon}\right)}\|\mathbf{g}\|_{H^{1 / 2}(\partial \Omega)}, \text { for } 1<t .
$$


Hence (9.20) is replaced by

$$
|\mathbf{u}|_{H^{1}(\Omega)} \leq C R^{t / 2}\|\mathbf{g}\|_{H^{1 / 2}(\partial \Omega)}^{1+t / 2}, \text { for } 1<t .
$$

Similarly, the statement of Theorem 10.3 holds provided $h_{b}<\frac{C}{R^{t}}$ for some $t>1$, and its conclusion is

$$
\left|\mathbf{u}_{h}\right|_{H^{1}(\Omega)} \leq C R^{t^{\prime} / 2}\|\mathbf{g}\|_{H^{1 / 2}(\partial \Omega)}^{1+t^{\prime} / 2}, \text { for } 1<t<t^{\prime},
$$

with a constant $C$ that is independent of $R, h$ and $\mathbf{g}$.

\section{ACKNOWLEDGMENTS}

The authors are grateful to the anonymous referee who read this article thoroughly and made very interesting and helpful comments and suggestions.

\section{REFERENCES}

[1] Adams, R. A., Sobolev Spaces, New York: Academic Press, 1975. MR 56:9247

[2] Amrouche, C. and Girault, V., "Decomposition of vector spaces and application to the Stokes problem in arbitrary dimension," Czechoslovak Math. J. 44 (119), pp. 109-140 (1994). MR 95c:35190

[3] Arnold, D. N., Scott, L. R. and Vogelius, M., "Regular inversion of the divergence operator with Dirichlet boundary conditions on a polygon," Ann. Scuola Norm. Sup. Pisa Cl. Sci.Serie IV, XV, pp.169-192, (1988). MR 91i:35043

[4] Bernardi, C. and Girault, V., "A local regularization operator for triangular and quadrilateral finite elements," SIAM J. Numer. Anal., 35, 5, pp.1893-1916, (1998). MR 99g:65107

[5] Brenner, S. and Scott, L. R., The Mathematical Theory of Finite Element Methods, New York: Springer-Verlag, 1994. MR 95f:65001

[6] Ciarlet, P. G., The Finite Element Method for Elliptic Problems, Amsterdam: North-Holland, 1978. MR 58:25001

[7] Clément, P., "Approximation by finite element functions using local regularization," R.A.I.R.O. Analyse Numérique R-2, pp.77-84, (1975). MR 53:4569

[8] Dupont, T. and Scott, L. R., "Polynomial approximation of functions in Sobolev spaces," Math. Comp., 34, pp.441-463, (1980). MR 81h:65014

[9] Girault, V. and Raviart, P.-A., Finite Element Methods for the Navier-Stokes Equations. Theory and Algorithms, SCM 5, Berlin:Springer-Verlag, 1986. MR 88b:65129

[10] Grisvard, P., Elliptic Problems in Nonsmooth Domains, Pitman Monographs and Studies in Mathematics, 24, Boston, MA: Pitman, 1985. MR 86m:35044

[11] Hopf, E., "Über die Aufangswertaufgabe für die hydrodynamischen Grundgleichungen," Math. Nachr., 4, pp.213-231, (1951). MR 14:327b

[12] Leray, J., "Etude de diverses équations intégrales non linéaires et de quelques problèmes que pose l'hydrodynamique," J. Math. Pures Appl., 12, pp.1-82, (1933).

[13] Lions, J.-L., Quelques Méthodes de Résolution des Problèmes aux Limites Non Linéaires, Dunod, Paris, 1969. MR 41:4326

[14] Lions, J.-L. and Magenes, E., Problèmes aux Limites non Homogènes et Applications, I, Paris: Dunod, 1968. MR 40:512

[15] Morgan, J. W. and Scott, L. R., "A nodal basis for $C^{1}$ piecewise polynomials of degree $n \geq 5$," Math. Comp., 29, pp.736-740, (1975). MR 51:11930

[16] Nečas, J., Les Méthodes Directes en Théorie des Équations Elliptiques, Prague: Academia, 1967. MR 37:3168

[17] Scott, L. R. and Vogelius, M., "Norm estimates for a maximal right inverse of the divergence operator in spaces of piecewise polynomials," $M^{2} A N$ (formerly R.A.I.R.O. Analyse Numérique), 19, pp.111-143, (1985). MR 87i:65190

[18] Scott, L. R. and Zhang, S., "Finite element interpolation of nonsmooth functions satisfying boundary conditions," Math. Comp., 54, pp.483-493, (1990). MR 90j:65021

[19] Stein, E., Singular integrals and differentiability properties of functions, Princeton, NJ: Princeton University Press, 1970. MR 44:7280 
[20] Temam, R., Infinite-dimensional dynamical systems in mechanics and physics, Applied Mathematical Sciences 68, Berlin:Springer-Verlag, 1997. MR 98b:58056

[21] Verfürth, R. "Error estimates for some quasi-interpolation operators," M2AN Math. Model. Numer. Anal. 33, pp. 695-713 (1999). MR 2001a:65149

Laboratoire d’Analyse Numérique, Université Pierre et Marie Curie, 75252 Paris CEDEX 05, FRANCE

E-mail address: girault@ann.jussieu.fr

Department of Mathematics and the Computation Institute, University of Chicago, Chicago, Illinois 60637-1581

E-mail address: ridg@uchicago.edu 